\title{
Piercing of domain walls: new mechanism of gravitational radiation
}

\author{
Dmitri Gal'tsov, ${ }^{a, b}$ Elena Melkumova ${ }^{a}$ and Pavel Spirin ${ }^{a}$ \\ ${ }^{a}$ Faculty of Physics, Moscow State University, \\ 119991, Moscow, Russian Federation \\ ${ }^{b}$ Kazan Federal University, \\ 420008, Kazan, Russian Federation \\ E-mail: galtsov@phys.msu.ru, elenamelk@mail.ru, pspirin@physics.uoc.gr
}

ABSTRACT: Domain wall (DW) moving in media undergoes the friction force due to particle scattering. However certain particles are not scattered, but perforate the wall. As a result, the wall gets excited in the form of the branon wave, while the particle experiences an acceleration jump. This gives rise to generation of gravitational waves which we call "piercing gravitational radiation" (PGR). Though this effect is of higher order in the gravitational constant than the quadrupole radiation from the collapsing DWs, its amplitude is enhanced in the case of relativistic particles or photons because of absence of the velocity factor which is present in the quadrupole formula. We derive the spectral-angular distribution of PGR within the simplified model of the weakly gravitating particle-wall system in Minkowski space-time of arbitrary dimensions. Within this model the radiation amplitude is obtained analytically. The spectral-angular distribution of PGR in such an approach suffers from infrared and ultraviolet divergences as well as from collinear divergence in the case of a massless perforating particle. Different cut-off schemes appropriate in various dimensions are discussed. Our results are applicable both to cosmological DWs and to the braneworld models. PGR can be relevant in the infrared part of the spectrum of the relic gravitons where radiation from the collapsed DWs is damped.

Keywords: Classical Theories of Gravity, Black Holes, Brane Dynamics in Gauge Theories, Large Extra Dimensions

ARXIV EPRINT: 1711.01114 


\section{Contents}

1 Introduction 1

2 The setup 3

2.1 The model 4

2.2 Iteration scheme 5

3 First order dynamics $\quad 7$

3.1 Particle's motion $\quad 7$

3.2 Piercing layer 8

3.3 The RS2 setup 9

3.4 Deformation of domain wall 10

4 Gravitational radiation formula revisited $\quad 12$

$\begin{array}{ll}4.1 \text { The second order } & 12\end{array}$

$\begin{array}{lll}4.2 & \text { New derivation } & 14\end{array}$

$\begin{array}{lll}4.3 & \text { Polarization tensors } & 16\end{array}$

5 Radiation amplitudes $\quad \mathbf{1 8}$

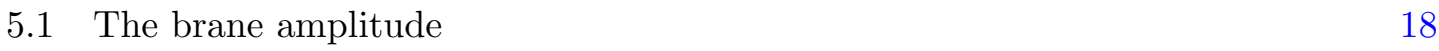

$\begin{array}{ll}5.2 & \text { The particle amplitude } \\ 5.3 & 20\end{array}$

5.3 The stress contribution 21

5.4 The destructive interference in the ultrarelativistic limit 22

$\begin{array}{ll}5.5 & \text { The massless case } \\ \end{array}$

6 The spectral and angular distribution of PGR 25

6.1 The ultra-relativistic case: beaming in the bulk direction 26

$\begin{array}{ll}6.2 & \text { Non-relativistic case: the brane contribution } \\ \end{array}$

$\begin{array}{lll}7 & \text { Conclusions } & 32\end{array}$

A Angular integration $\quad 34$

B Applicability of the perturbation theory 36

\section{Introduction}

Since their prediction by Zeldovich, Kobzarev and Okun [1, 2] and Kibble [3], cosmological DWs remain in the focus of theoretical study for more than forty years. DWs are formed during the phase transitions in the early universe once the discrete symmetry of the underlying gauge theory is spontaneously broken. After creation, their average number per a Hubble radius remains constant for some time, so, if they were stable, their energy density potentially could dominate and overclose the Universe [4-7]. To avoid this, DWs 
either must be unstable, what happens if the discrete symmetry was only approximate, or disappear via some other mechanism. The basic viable field model of cosmological DWs is that of real scalar field with the biased potential [8]. Collapsing unstable DWs generate gravitational waves, whose spectrum, sensible to particular underlying models, can be an important source of information about the early universe. In view of the forthcoming experimental studies of relict gravitational waves, this subject attracted attention recently [8-14], for a review see [15]. Particular models considered include the hybrid inflation [9], the standard model extended to the very early universe [14], the Higgs model [11], the next-to-minimal supersymmetric standard model [12] and some other. The results are based on numerical simulations of creation and annihilation of DW with account for gravitational radiation computed via the correlation functions of the energy-momentum tensors. These are in good agreement with the simple estimates based on the quadrupole formula for gravitational radiation of non-relativistic systems [13].

Collapsing DWs emit gravitational radiation due to their intrinsic dynamics, so the above mentioned numerical simulations seem to give major contribution to graviton production from the motion of the unstable DWs. These calculations, however, do not take into account gravitational interaction of DW with surrounding matter which may lead to additional generation of gravitational waves, maybe subdominant, but with distinct spectral properties. This may be especially important if one assumes that the spontaneously broken discrete symmetry was exact, so no intrinsic instability of DWs is supposed. Here we would like to discuss one such a mechanism which may be considered as generalization of the gravitational bremsstrahlung in particle collisions. We will be interested in perforating collisions of particles with extended objects. For more generality, we consider this process in any dimensions keeping in mind also the braneworld scenarios of the Randall-Sundrum II type [16-19]. There the DW corresponds to factoring of the AdS5 geometry which leads to a particularly simple analytical description.

Recall that punctures of DWs, producing holes inside them, may serve as an alternative mechanism of DW destruction [20]. In the models admitting both the DWs and the cosmic strings, the hole of finite size in the wall must be surrounded by the string. Formation of such a hole changes the overall energy balance forcing the hole either to shrink due to tension of the string, or to expand due to DW tension, eventually eating the wall. More precisely, as was shown in [20], at least four holes are needed for the second option to be realized. The holes in the DWs could be created by bulk black holes perforating them [21], so physics of perforation is worth to be explored in detail. The field-theoretical treatment of the collision of black holes with DWs was developed in [22]. These effects can be regarded as topological phase transitions. Other aspects of such transitions in the composite braneblack hole systems were studied in [19, 23-27]. In the context of the braneworld models, the interaction of black holes with DWs attracted much attention in connection with the conjectured creation of black holes in particle collisions on the brane and their possible escape into the bulk [28-30]. (In this framework we prefer to use the term "brane", though we always deal with branes of co-dimension one, that is with DWs.)

The problem considered here, though is related to the above issues, is, however, somewhat different. We discuss perforation of DWs by elementary particles of the surround- 
ing plasma (or bulk particles in the braneworld case) which classically have zero size, so our effects rather are dynamical than topological. We use the framework first suggested in [31] and further developed in [32-34], which essentially consists of perturbative description of gravitational interaction of the Nambu-Goto DW and the classical point particle in Minkowski space. This approach opens a way to describe analytically the branon excitation of the wall revealing creation of the free branon wave. This phenomenon was first found within the RS II five-dimensional model [31] and then generalized to arbitrary dimensions [32] showing that the branon effects are dimension-dependent in view of the different causal structure of propagators of massless fields in even and odd space-time dimensions [35-37]. The particle-DW interaction has the growing potential, so there are no free asymptotic states. Still it turned out possible to introduce the dressed momenta for both objects which are instantaneously conserved during the collision [33]. We also calculated emission of scalar waves under perforation assuming that the particle (but not the wall) to interact with a scalar field apart of gravity [34].

Here we calculate genuine gravitational radiation from the same system suggesting this as new and mechanism of radiation: "piercing gravitational radiation" (PGR), with some novel conceptual and technical features. First, the system has no wave zone. This prompted us to revisit derivation of the spectral-angular distribution of radiated energy not referring to wave zone. Second, the effective radiating current contains a light-like part (due to the free branon) with associated problems in constructing the retarded solutions of the D'Alembert equation with light-like sources [38-40]. Furthermore, radiation exhibits peculiar infrared and collinear [41-44] divergences, typical for radiation from massless charges in gauge theories (for more detailed discussion see [45]). Recently, these matters were extensively studied in connection with the memory effect [40, 46-49] and the Bondi-Metzner-Sachs asymptotic symmetries [50]. Though we do not discuss these very interesting subjects here, we feel that our radiation problem provides a novel interesting setting for these studies too.

Physically, PGR may be relevant for cosmic DWs, providing additional low-frequency contributions to the standard spectrum of gravitons from collapsing walls, which is typically dies off in the infrared [15]. Detailed study of such applications, however, remains beyond the scope of the present paper which is mostly restricted to theoretical aspects of the problem.

\section{The setup}

We consider the gravitating system of an infinite Nambu-Goto DW of plain topology and a point particle. Omitting the self-gravity of each object, we treat the full metric generated by them via Einstein equations and the motion of both objects in this metric self-consistently in the framework of the perturbation theory on the Minkowski background in terms of the coupling constant $\varkappa\left(\varkappa^{2}=16 \pi G_{D}\right)$, where $G_{D}$ is the $D$-dimensional Newton constant (we use the units $c=1$ ). When gravity is switched off, the following geometry of the collision is assumed: the plane infinite Nambu-Goto brane sits at rest in $D$-dimensional Minkowski space-time, so that its world-volume is orthogonal to $z$-axis. A point particle of 
mass $m$ is moving along $z$-axis with some initial velocity such that it reaches the wall and perforates it. The $D$-dimensional cartesian coordinates are therefore split as $x^{M}=\left(x^{\mu} ; z\right)$, $x^{\mu} \in M_{1, D-2}$, the metric signature is $(+,-, \ldots,-)$. Our conventions for the Riemann and Ricci tensors are: $R^{B}{ }_{N R S} \equiv \Gamma_{N S, R}^{B}-\Gamma_{N R, S}^{B}+\Gamma_{N S}^{A} \Gamma_{A R}^{B}-\Gamma_{N R}^{A} \Gamma_{A S}^{B}$ and $R_{M N} \equiv \delta_{A}^{B} R_{M B N}^{A}$.

\section{$2.1 \quad$ The model}

We keep notation introduced in the previous papers [32-34]. The $(D-2)$-dimensional DW propagating in the $D$-dimensional space-time $\mathcal{M}_{D}$ with the metric $g_{M N}$, has the worldvolume $\mathcal{V}_{D-1}$ parametrized by arbitrary coordinates $\sigma^{\mu}, \mu=0,1,2, \ldots, D-2$ and defined by the embedding equations $x^{M}=X^{M}\left(\sigma^{\mu}\right), M=0,1,2, \ldots, D-1$. The point mass propagates normally to the DW along the worldline $x^{M}=z^{M}(\tau)$, affinely parametrized and described by the Polyakov action using the einbein $e(\tau)$. To total action governing the system reads

$$
\begin{aligned}
S= & -\frac{\mu}{2} \int\left[X_{\mu}^{M} X_{\nu}^{N} g_{M N} \gamma^{\mu \nu}-(D-3)\right] \sqrt{-\gamma} d^{D-1} \sigma-\frac{1}{2} \int\left(e g_{M N} \dot{z}^{M} \dot{z}^{N}+\frac{m^{2}}{e}\right) d \tau- \\
& -\frac{1}{\varkappa^{2}} \int R_{D} \sqrt{-g} d^{D} x .
\end{aligned}
$$

Here $\mu$ denotes the brane tension, $X_{\mu}^{M}=\partial X^{M} / \partial \sigma^{\mu}$ are the tangent vectors on the DW world-volume and $\gamma^{\mu \nu}$ is the inverse metric on it, $\gamma=\operatorname{det} \gamma_{\mu \nu}$.

Variation of (2.1) with respect to $X^{M}$ gives the brane equation of motion in the covariant form

$$
\partial_{\mu}\left(X_{\nu}^{N} g_{M N} \gamma^{\mu \nu} \sqrt{-\gamma}\right)=\frac{1}{2} g_{N P, M} X_{\mu}^{N} X_{\nu}^{P} \gamma^{\mu \nu} \sqrt{-\gamma},
$$

while variation with respect to $\gamma^{\mu \nu}$ gives the constraint equation

$$
\left(X_{\mu}^{M} X_{\nu}^{N}-\frac{1}{2} \gamma_{\mu \nu} \gamma^{\lambda \tau} X_{\lambda}^{M} X_{\tau}^{N}\right) g_{M N}+\frac{D-3}{2} \gamma_{\mu \nu}=0
$$

whose solution defines $\gamma_{\mu \nu}$ as the induced metric on $\mathcal{V}_{D-1}$ :

$$
\gamma_{\mu \nu}=\left.X_{\mu}^{M} X_{\nu}^{N} g_{M N}\right|_{x=X}
$$

Varying $S$ with respect to $e(\tau)$ and $z^{M}(\tau)$ one obtains the equations

$$
e^{2} g_{M N} \dot{z}^{M} \dot{z}^{N}=m^{2}
$$

and

$$
\frac{d}{d \tau}\left(e \dot{z}^{N} g_{M N}\right)=\frac{e}{2} g_{N P, M} \dot{z}^{N} \dot{z}^{P}
$$

respectively, while variation over $g_{M N}$ leads to the Einstein equations

$$
G^{M N}=\frac{1}{2} \varkappa^{2}\left[T^{M N}+\bar{T}^{M N}\right],
$$


with

$$
\begin{aligned}
& T^{M N}=\mu \int X_{\mu}^{M} X_{\nu}^{N} \gamma^{\mu \nu} \frac{\delta^{D}(x-X(\sigma))}{\sqrt{-g}} \sqrt{-\gamma} d^{D-1} \sigma, \\
& \bar{T}^{M N}=e \int \frac{\dot{z}^{M} \dot{z}^{N} \delta^{D}(x-z(\tau))}{\sqrt{-g}} d \tau
\end{aligned}
$$

for the energy-momentum tensor of the brane and a particle, respectively. Eventually in our expansions we will use Einstein equations with lowercase indices.

Though the infinitely thin DW is still compatible with the full non-linear gravity, the point-like particle is not. But it sensible to consider our system in the context of the perturbative gravity on Minkowski background. In this approach one presents the metric as

$$
g_{M N}=\eta_{M N}+\varkappa h_{M N},
$$

and expands all quantities in powers of $\varkappa$, making use of $\eta_{M N}$ to raise and to lower the indices. It is convenient to define the quantity

$$
\psi_{M N} \equiv h_{M N}-\frac{h}{2} \eta_{M N}, \quad h \equiv \eta^{M N} h_{M N},
$$

and to choose the flat-space harmonic gauge (in all orders in $\varkappa$ )

$$
\partial_{N} \psi^{M N}=0 .
$$

\section{$2.2 \quad$ Iteration scheme}

Like in the particle scattering problem, we use the simultaneous expansions of the particle world-line $z^{M}(\tau)$, the DW embedding functions $X^{M}\left(\sigma^{\mu}\right)$ and the metric deviation $h_{M N}$ in $\varkappa$. It is understood that in zero order the particle moves freely along the line orthogonal to the wall and pierces it. The subtle point of this setting is that actually the gravitational potential of the Nambu-Goto plane wall is growing in space, so the system has no free asymptotic states at all. Consequently one can not define the momentum of the particle in the initial state. This gives rise to complications in establishing the momentum balance equation. However, as was discussed in our previous paper [33], there is a way out in constructing the "dressed" momenta, so we will not enter into this here. We still can use the formal expansions of the unknown functions in the gravitational constant keeping in mind that we are actually considering a vicinity of the wall whose size is small with respect to curvature radius of an exact DW metric. Gravitational field of DW is repulsive, so to be able to pierce the DW, the particle must have enough energy in order not to be reflected. We will see in what follows that for any particle velocity there is some domain from which the particles reach DW. We will call this velocity dependent domain the piercing layer, its size will be defined in the section $2 \mathrm{C}$. So actually our iterative scheme applies only to the piercing layer.

With this in mind, we proceed now with formal expansions

$$
\Phi={ }^{0} \Phi+{ }^{1} \Phi+{ }^{2} \Phi+\ldots,
$$


where $\Phi$ denotes collectively the set of variables: $z^{M}(\tau), e(\tau), X^{M}(\sigma)$ and $h_{M N}(x)$. The left superscript labels the order in terms $\varkappa$.

The zeroth order is trivial. It describes a free plane unperturbed brane and a particle moving with constant velocity $\left(u^{M}=\gamma(1,0, \ldots, 0, v)\right)$ perpendicular to the brane where $\gamma=1 / \sqrt{1-v^{2}}$ :

$$
{ }^{0} z^{M}(\tau)=u^{M} \tau
$$

in the absence of the gravitational field $h_{M N}=0$. The Lagrange multiplier $e$ is chosen to be equal to the corresponding particle mass ${ }^{0} e=m$, so that the trajectories are parametrized by proper time and the velocity satisfies the normalization condition $\eta_{M N} u^{M} u^{N} \equiv u^{2}=1$.

In the zeroth order in $\varkappa$ the brane is assumed to be unexcited

$$
{ }^{0} X^{M}=\Sigma_{\mu}^{M} \sigma^{\mu},
$$

where $\Sigma_{\mu}^{M}$ are $(D-1)$ constant bulk Minkowski vectors which can be normalized as

$$
\Sigma_{\mu}^{M} \Sigma_{\nu}^{N} \eta_{M N}=\eta_{\mu \nu}
$$

so the corresponding induced metric on $\mathcal{V}_{D-1}$ is flat. Obviously, this is a solution to the eq. (2.2) for $\varkappa=0$, and the corresponding induced metric is four-dimensional Minkowski metric $\gamma_{\mu \nu}=\eta_{\mu \nu}$. Thus it is convenient with no loss of generality to fix

$$
\Sigma_{\mu}^{M}=\delta_{\mu}^{M} .
$$

In other words, we choose the Lorentz frame where the unperturbed brane is at rest.

The first order correction is obtained next. The zeroth order straight particle trajectory and the flat brane are sources of the first order gravitational field ${ }^{1} h_{M N}^{\mathrm{p}} \equiv \bar{h}_{M N}$ of the particle and ${ }^{1} h_{M N}^{\mathrm{br}} \equiv h_{M N}$ of the brane, respectively:

$$
{ }^{1} h_{M N}=h_{M N}+\bar{h}_{M N} .
$$

In turn, $h_{M N}$ causes the first order deviation of the trajectory ${ }^{1} z^{M}$, while $\bar{h}_{M N}$ produces the first order deviation ${ }^{1} X^{\mu}(\sigma)$ of the embedding functions. In the process, the first correction ${ }^{1} e$ of the einbein fields is also obtained. Explicitly, from the zeroth order trajectories one obtains the zeroth order particle energy-momentum tensor

$$
{ }^{0} \bar{T}^{M N}=m \int u^{M} u^{N} \delta^{D}\left(x-{ }^{0} z(\tau)\right) d \tau,
$$

which in this order has only $t, z-$ components, and from the first order Einstein equations for particle field, given by

$$
\square \bar{h}^{M N}=-\varkappa\left({ }^{0} \bar{T}^{M N}-\frac{1}{D-2}{ }^{0} \bar{T} \eta^{M N}\right),
$$

the first order correction ${ }^{1} h^{M N}$ to the metric is obtained. In what follows, the stress-tensor and the gravitational field of the particle will be denoted by bar. 
Passing to the $D$-dimensional Fourier-transforms

$$
\bar{h}_{M N}(x)=\frac{1}{(2 \pi)^{D}} \int \mathrm{e}^{-i q x} \bar{h}_{M N}(q) d^{D} q, \quad \bar{T}^{M N}(x)=\frac{1}{(2 \pi)^{D}} \int \mathrm{e}^{-i q x} \bar{T}^{M N}(q) d^{D} q,
$$

we obtain the retarded solution in the momentum representation

$$
\bar{h}_{M N}(q)=\frac{2 \pi \varkappa m \delta(q u)}{q^{2}+i \varepsilon q^{0}}\left(u_{M} u_{N}-\frac{1}{D-2} \eta_{M N}\right) .
$$

In the coordinate representation we find (for $D \geqslant 4$ ):

$$
\bar{h}_{M N}(x)=-\frac{\varkappa m \Gamma\left(\frac{D-3}{2}\right)}{4 \pi^{\frac{D-1}{2}}}\left(u_{M} u_{N}-\frac{1}{D-2} \eta_{M N}\right) \frac{1}{\left[\gamma^{2}(z-v t)^{2}+r^{2}\right]^{\frac{D-3}{2}}},
$$

where $r=\sqrt{\delta_{i j} \sigma^{i} \sigma^{j}}$ is the radial distance on the wall from the perforation point. This is just the Lorentz-contracted $D$-dimensional Newton field of the uniformly moving particle.

The zeroth-order expression for the brane energy-momentum tensor $T^{M N}$ reads:

$$
{ }^{0} T^{M N}=\mu \int \Sigma_{\mu}^{M} \Sigma_{\nu}^{N} \eta^{\mu \nu} \delta^{D}\left(x-{ }^{0} X(\sigma)\right) d^{D-1} \sigma,
$$

so the Einstein equation for the first-order brane field is given by:

$$
\square h^{M N}=-\varkappa\left({ }^{0} T^{M N}-\frac{1}{D-2}{ }^{0} T \eta^{M N}\right), \quad{ }^{0} T \equiv{ }^{0} T^{M N} \eta_{M N} .
$$

The Fourier-space solution is given by

$$
h_{M N}(q)=\frac{(2 \pi)^{D-1} \varkappa \mu}{q^{2}}\left(\Xi_{M N}-\frac{D-1}{D-2} \eta_{M N}\right) \delta^{D-1}\left(q^{\mu}\right),
$$

where $\Xi_{M N} \equiv \Sigma_{M}^{\mu} \Sigma_{N}^{\nu} \eta_{\mu \nu}$, while in the coordinate space it reads

$$
h_{M N}=\frac{\varkappa \mu}{2}\left(\Xi_{M N}-\frac{D-1}{D-2} \eta_{M N}\right)|z|=\frac{\varkappa \mu|z|}{2(D-2)} \operatorname{diag}(-1,1, \ldots, 1, D-1) .
$$

\section{First order dynamics}

\subsection{Particle's motion}

Using ${ }^{1} h^{M N}$ and the zeroth order solution in equations (2.4) and (2.5) one obtains for ${ }^{1} e$ and ${ }^{1} z^{M}$ the equations ${ }^{1}$

$$
{ }^{1} e=-\frac{m}{2}\left(\varkappa h_{M N} u^{M} u^{N}+2 \eta_{M N} u^{M 1} \dot{z}^{N}\right)
$$

and

$$
\frac{d}{d \tau}\left({ }^{1} e u_{M}+m^{1} \dot{z}_{M}\right)=-\varkappa m\left(h_{P M, Q}-\frac{1}{2} h_{P Q, M}\right) u^{P} u^{Q},
$$

\footnotetext{
${ }^{1}$ Our gauge condition is $g_{M N} \dot{z}^{M} \dot{z}^{N}=1$. To this order it reduces to ${ }^{1} e=0$.
} 
which upon elimination of ${ }^{1} e$ give

$$
1 \ddot{z}_{M}=-\varkappa\left(h_{P M, Q}-\frac{1}{2} h_{P Q, M}\right) u^{P} u^{Q},
$$

or, in the components,

$$
{ }^{1} \ddot{z}^{0}=2 k v \gamma^{2} \operatorname{sgn}(\tau), \quad \delta \ddot{z} \equiv{ }^{1} \ddot{z}^{D-1}=k\left(D \gamma^{2} v^{2}+1\right) \operatorname{sgn}(\tau),
$$

where a parameter is introduced

$$
k=\frac{\mu \varkappa^{2}}{4(D-2)}
$$

playing the role of the inverse bulk curvature radius in the full non-linear treatment. According to (3.4) the gravity force between the DW and the particle is repulsive. (Recall that this "antigravity" is caused by the dominance of the DW tension (negative pressure) in the energy-momentum tensor of the wall.)

Integrating (3.4) twice with initial conditions $\delta z^{M}(0)=0, \delta \dot{z}^{M}(0)=0$, one has

$$
{ }^{1} z^{0}=k v \tau^{2} \gamma^{2} \operatorname{sgn}(\tau), \quad \delta z=\frac{1}{2} k \tau^{2}\left(D \gamma^{2} v^{2}+1\right) \operatorname{sgn}(\tau) .
$$

Substituting (3.5) into (3.1) one can check that the gauge condition ${ }^{1} e=0$ is satisfied.

\subsection{Piercing layer}

We now discuss physical restrictions of our iterative scheme in more detail. Suppose that a point particle moving in the linearized gravitational field of the brane passes through the point $z_{l}>0$ with the velocity $d z / d t=v<0$. According to eq. (3.4) it has a (proper) acceleration $\ddot{z}=a=k\left(D \gamma^{2} v^{2}+1\right)$. This particle reaches the brane at the proper time moment

$$
\tau_{0}=\frac{|v| \gamma}{k\left(D \gamma^{2} v^{2}+1\right)}\left(1-\sqrt{1-\frac{2 k z_{l}\left(D \gamma^{2} v^{2}+1\right)}{v^{2} \gamma^{2}}}\right) .
$$

With account for the reflection symmetry, it is clear that if $|z|>z_{l}$, where

$$
z_{l}(v)=\frac{v^{2} \gamma^{2}}{2 k\left(D \gamma^{2} v^{2}+1\right)},
$$

the particle will be reflected, while if $|z|<z_{l}$ it reaches the brane and passes through it. Thus the eq. (3.7) defines the boundary of the velocity-dependent layer of particles which pierce the brane. Or, conversely, for given $z$, only those particles which have the velocity $v>v_{l}(z)$, where $v_{l}(z)$ is the inverse function to $z_{l}(v)$, namely,

$$
v_{l}(z)=\frac{1}{\gamma} \sqrt{\frac{2 k z}{1-2 k z D}}
$$

will reach DW and pierce it.

We will see in the next subsection that the parameter $k$ defines the curvature of the DW gravitational field. The linearized metric of the wall is correct if $k z \ll 1$ [32]. In 
the non-relativistic case one has $k z_{l}=v^{2} / 2$, so our approximation is consistent and the piercing layer is small in the units $k^{-1}$. In the ultrarelativistic case $k z_{l}=(2 D)^{-1}$, so the layer size is of the order of the inverse curvature. In this case the condition of validity of the linearized approximation for the brane metric is only marginally satisfied.

Formally, the unperturbed motion is free in our scheme, but one has to keep in mind that the validity of this description is restricted to particles in the piercing layer only. Since this requirement is imposed a posteriori, one can expect that formal application of such an approach will face certain problems. Indeed, as we will see, the spectrum of gravitational waves will require cut-offs to get finite results.

\subsection{The RS2 setup}

The piercing layer can be described using the full non-linear treatment as well. A convenient setup is the one-brane Randall-Sundrum model (RS II) [16] adapted to arbitrary dimensions. Note that geodesic motion in the RS II setup was earlier considered with different motivation in [51-56]. ${ }^{2}$ The metric of the RS II model reads:

$$
\mathrm{d} s^{2}=\mathrm{e}^{-2 k\left|z_{R S}\right|} \mathrm{d} s_{\mathcal{M}}^{2}-\mathrm{d} z_{R S}^{2},
$$

where $\mathrm{d} s_{\mathcal{M}}^{2}$ is the flat metric on the brane. At the distance small compared with the curvature radius of the AdS bulk, $k z_{R S} \ll 1$, so that $\mathrm{e}^{-2 k\left|z_{R S}\right|} \simeq 1-2 k\left|z_{R S}\right|$ we see that $z_{R S}$ differs from our previous $z$ by a coordinate transformation. Indeed, the gauge for the $\mathrm{RS}$ solution is non-harmonic. To pass to the harmonic gauge used above one writes

$$
z_{R S}=z-2 k z^{2} \operatorname{sign}(z)
$$

reproducing the linearized metric (2.14). Note that this transformation is non-singular on the brane: $\partial z_{R S} / \partial z=1$ at $z=0$. For brevity, we omit an index in $z_{R S}$ in the rest of this section.

Using the non-vanishing Christoffel symbols

$$
\Gamma_{i j}^{0}=-\mathrm{e}^{-2 k|z|} k \eta_{i j} \operatorname{sgn} z \quad \Gamma_{j z}^{i}=-k \delta_{j}^{i} \operatorname{sgn} z
$$

in the geodesic equation $\ddot{x}^{M}+\Gamma_{L R}^{M} \dot{x}^{L} \dot{x}^{S}=0$ one derives the following two equations

$$
\ddot{t}=2 k \dot{t} \dot{z} \operatorname{sgn} z, \quad \ddot{z}=\mathrm{e}^{-2 k|z|} k \dot{t}^{2} \operatorname{sgn} z,
$$

whose solution is

$$
z(t)= \pm \frac{1}{2 k} \ln \left(k^{2} t^{2}+\mathrm{e}^{2 k\left|z_{0}\right|}\left[1 \mp 2 k v_{0} t\right]\right),
$$

where $v_{0}=-(d z / d t)_{t=0}$. The time $T$ needed for the particle at an initial distance $l$ to reach the brane is

$$
T=\frac{1}{k}\left[\mathrm{e}^{2 k l} v_{0}-\sqrt{e^{4 k l} v_{0}^{2}-\mathrm{e}^{2 k l}+1}\right],
$$

\footnotetext{
${ }^{2}$ Actually the bulk in the RS II model is anti-deSitter, and the negative cosmological constant is present, but for our local considerations restricted by the vicinity of the brane this is irrelevant.
} 
so we find the following condition of piercing: the lowest initial value of the velocity $v_{0}$ has to be

$$
v_{0}^{\min }=\mathrm{e}^{-2 k l} \sqrt{\mathrm{e}^{2 k l}-1} .
$$

As a function of the product $k l$, the velocity $v_{0}^{\min }$ has the maximum $1 / 2$ at $k l=\ln 2 / 2$. Hence, if $v_{0}>1 / 2$, the particle reaches the brane independently of the initial distance. Conversely, for the fixed initial velocity $v_{0}$, the largest initial distance $l^{\max }$ is

$$
l^{\max }=\frac{1}{2 k} \ln \left(\frac{1-\sqrt{1-4 v_{0}^{2}}}{2 v_{0}^{2}}\right), \quad v_{0}<\frac{1}{2} .
$$

Thus in our problem the non-relativistic limit implies either the Minkowski limit of the metric, or an initial particle position on the brane. In the non-relativistic limit one has

$$
l^{\max } \simeq \frac{v_{0}^{2}}{2 k}
$$

which reproduces the result of the previous subsection (3.7) taken in the non-relativistic approximation. The particle velocity at the moment of piercing is given by

$$
\left.v_{\mathrm{br}} \equiv \frac{d z}{d t}\right|_{t=T}=-\sqrt{e^{4 k l} v_{0}^{2}-\mathrm{e}^{2 k l}+1},
$$

so the condition of applicability of the perturbation theory is

$$
\frac{v_{0}-\left|v_{\mathrm{br}}\right|}{v_{0}} \ll 1
$$

Being translated to the possible values of $k l$, this gives

$$
k l \ll 1,
$$

so

$$
\frac{v_{0}-\left|v_{\mathrm{br}}\right|}{v_{0}} \simeq k l\left(2-\frac{1}{v_{0}^{2}}\right)
$$

and $k l \ll 1$ is sufficient for the validity of our iterative scheme. Hence, expanding $T$ (3.12) in powers of $k$ one obtains

$$
T \simeq \frac{l}{v_{0}}[1+\mathcal{O}(k l)]
$$

what corresponds to the linear gravity.

\subsection{Deformation of domain wall}

Now we consider perturbations of DW due to gravitational interaction with the particle. For this we have to use the metric deviation due to the particle. In accordance with our iterative scheme we neglect particle's acceleration in the wall gravity when we calculate its proper gravitational field, considering the unperturbed particle trajectory. 
Perturbations of the Nambu-Goto branes in the external gravitational field were expensively studied in the past, see e.g. $[57,58]$. The derivation is particularly simple in the Minkowski background . First, from eq. (2.3) we find the perturbation of the induced metric

$$
\delta \gamma_{\mu \nu}=2 \Sigma_{(\mu}^{M} \delta X_{\nu)}^{N} \eta_{M N}+\varkappa \bar{h}_{M N} \Sigma_{\mu}^{M} \Sigma_{\nu}^{N}
$$

where brackets denote symmetrization over the indices with a factor $1 / 2$. Linearizing the rest of the eq. (2.2), after some rearrangements one obtains the following equation for the deformation of the wall:

$$
\Pi_{M N} \square_{D-1} \delta X^{N}=\Pi_{M N} J^{N}, \quad \Pi^{M N} \equiv \eta^{M N}-\Sigma_{\mu}^{M} \Sigma_{\nu}^{N} \eta^{\mu \nu},
$$

where $\square_{D-1} \equiv \partial_{\mu} \partial^{\mu}$ and $\Pi^{M N}$ is projector onto the (one-dimensional) subspace orthogonal to $\mathcal{V}_{D-1}$. The source term in (3.17) reads:

$$
J^{N}=\varkappa \Sigma_{P}^{\mu} \Sigma_{Q}^{\nu} \eta_{\mu \nu}\left(\frac{1}{2} \bar{h}^{P Q, N}-\bar{h}^{N P, Q}\right)_{z=0} .
$$

Using the aligned coordinates on the brane $\sigma^{\mu}=(t, \mathbf{r})$, we will have $\delta_{\mu}^{M}=\Sigma_{\mu}^{M}$, so the projector $\Pi^{M N}$ reduces the system (3.17) to a single equation for the $M=z$ component. Thus only the $z$-component of $\delta X^{M}$ and $J^{M}$ is physical. Generically, the transverse deformations of branes can be viewed as Nambu-Goldstone bosons (branons) which result from spontaneous breaking of the translational symmetry [59]. In the brane-world models these are coupled to matter on the brane via the induced metric (for a recent discussion see $[60,61])$. In our case of co-dimension one there is only one such branon. The remaining components of the perturbation $\delta X^{M}$ can be removed by transformation of the coordinates on the world-volume, so $\delta X^{\mu}=0$ is nothing but the gauge choice. Note that in this gauge the perturbation of the induced metric $\delta \gamma_{\mu \nu}$ does not vanish, contrary to the perturbation of the particle ein-bein $e$.

Denoting the physical component as $\Phi\left(\sigma^{\mu}\right) \equiv \delta X^{z}$ we obtain the branon $(D-1)$ dimensional wave equation:

$$
\eta^{\mu \nu} \frac{\partial}{\partial \sigma^{\mu}} \frac{\partial}{\partial \sigma^{\nu}} \Phi(\sigma)=J(\sigma),
$$

with the source term $J \equiv J^{z}$. Substituting (2.12) into the eq. (3.18) we obtain the source term for the branon:

$$
J(\sigma)=-\varkappa\left[\frac{1}{2} \eta_{\mu \nu} \bar{h}^{\mu \nu, z}-\bar{h}^{z 0,0}\right]_{z=0}=-\frac{\lambda v t}{\left[\gamma^{2} v^{2} t^{2}+r^{2}\right]^{\frac{D-1}{2}}},
$$

where

$$
\lambda=\frac{\varkappa^{2} m \gamma^{2} \Gamma\left(\frac{D-1}{2}\right)}{4 \pi^{\frac{D-1}{2}}}\left(\gamma^{2} v^{2}+\frac{1}{D-2}\right) .
$$

Construction of the retarded solution of the eq. (3.19) was explained in [31, 32], the result consists of two terms:

$$
\Phi(t, \mathbf{r})=-\Lambda \operatorname{sgn}(t) I_{\mathrm{a}}+2 \Lambda \theta(t) I_{\mathrm{b}}, \quad \Lambda \equiv \frac{\sqrt{\pi} \lambda}{2^{\frac{D-2}{2}} \gamma^{3} \Gamma\left(\frac{D-1}{2}\right)},
$$


with

$$
\begin{aligned}
& I_{\mathrm{a}}(t, r)=\frac{1}{r^{\frac{D-4}{2}}} \int_{0}^{\infty} d y J_{\frac{D-4}{2}}(y r) y^{\frac{D-6}{2}} \mathrm{e}^{-y \gamma v|t|}, \\
& I_{\mathrm{b}}(t, r)=\frac{1}{r^{\frac{D-4}{2}}} \int_{0}^{\infty} d y J_{\frac{D-4}{2}}(y r) y^{\frac{D-6}{2}} \cos y t,
\end{aligned}
$$

from which the first describes the odd in time brane deformation caused by the Lorentzcontracted Newton field of the particle, while the second is the shock branon wave arising at the moment of perforation and then freely propagating outwards along the brane. For $D=4$ these two integrals diverge logarithmically, though, as it was shown in [32], the corresponding regularized solutions exist. Here we will use the direct solution of the eq. (3.19) in the momentum representation:

$$
\Phi(q)=-\frac{i \pi \varkappa^{2} m}{\gamma} \frac{q^{z} \delta\left(q^{0}-v q^{z}\right)}{q_{M} q^{M}\left(q_{\mu} q^{\mu}+2 i \epsilon q^{0}\right)}\left(\gamma^{2} v^{2}+\frac{1}{D-2}\right) .
$$

\section{Gravitational radiation formula revisited}

Traditionally, both electromagnetic and gravitational radiation is computed in terms of fluxes of the field momentum in the wave zone, which is well-defined only in the asymptotically flat space-time. Our space-time is not asymptotically flat, so one should revisit the derivation. In particular, the energy-momentum flux through the lateral surface of the world-tube turns out to be non-zero [34]. Meanwhile, one can transform the flux at infinity, when it is well defined, into the volume integral extended through the space-time. This allows one to express the radiation power as an integral over the graviton momentum from the square of the source stress-tensor in the momentum representation contracted with polarization tensors [62]. Here we present the derivation of essentially the same formula without reference to the wave zone.

\subsection{The second order}

In the second order in $\varkappa$ one obtains the leading contribution to gravitational radiation. Actually, the source of radiation consists of three ingredients. The first is due to the particle which has constant acceleration before and after piercing. This has certain analogy with the Weinberg's approach [62] to compute gravitational radiation from the system of particles colliding at a point: in that case one has the constant momenta before and after collision which instantaneously change on a finite amount. In our case it is the (proper) time derivatives of the momenta before and after collision which are constant and opposite, changing sign at the moment of perforation. The second contribution comes from the deformation of the brane world-volume caused by varying gravitational field of the moving particle. Recall, that in our setting the brane is plane and non-excited once gravitational interaction is switched off. Finally, for consistency of calculations, the gravitational stresses have to be taken into account, these are described using Weinberg's expansion of the 
Einstein tensor up to the second order in the gravitational constant [62]. The gravitational stresses constitute the third component of the source.

The Einstein equation expanded up to the second order together with the corresponding expansion of the metric leads to the following equation for the second-order (tracereversed) metric deviation: ${ }^{2} \psi_{M N}$ :

$$
{ }^{2} \psi_{M N}=-\varkappa \tau_{M N},
$$

with the source containing three terms:

$$
\tau_{M N}={ }^{1} \bar{T}_{M N}+{ }^{1} T_{M N}+S_{M N} .
$$

The first is the particle term following from the eq. $(2.7):^{3}$

$$
\begin{aligned}
{ }^{1} \bar{T}_{M N}(x)=m \int & {\left[2{ }^{1} \dot{z}_{(M} u_{N)}+\varkappa\left(2 u^{P} h_{P(M} u_{N)}-\frac{h}{2} u_{M} u_{N}\right)-u_{M} u_{N}{ }^{1} z^{P} \partial_{P}\right] } \\
& \times \delta^{D}\left(x-{ }^{0} z(\tau)\right) d \tau .
\end{aligned}
$$

The second term ${ }^{1} T_{M N}$ represents the brane contribution. To compute it, one substitutes the first-order metric deviation (2.12) and the first-order brane perturbations into the eq. (2.7), keeping the quantities of the desired order:

$$
\begin{aligned}
{ }^{1} T_{M N}= & \frac{\mu}{2} \int\left[4 \Sigma_{(M}^{\mu}{ }^{1} X_{N)}^{\nu} \eta_{\mu \nu}+4 \bar{h}_{\lambda(M} \Sigma_{N)}^{\lambda}-2 \Sigma_{M}^{\mu} \Sigma_{N}^{\nu}\left(\bar{h}_{\mu \nu}+2 \eta_{L R} \Sigma_{(\mu}^{R}{ }^{1} X_{\nu)}^{L}\right)\right. \\
& \left.+\Sigma_{M}^{\mu} \Sigma_{N}^{\nu} \eta_{\mu \nu}\left(\bar{h}_{\lambda}^{\lambda}-\bar{h}+2{ }^{1} X_{\lambda}^{L} \Sigma_{L}^{\lambda}-2{ }^{1} X^{L} \partial_{L}\right)\right] \delta^{D}\left(x^{A}-\Sigma_{\alpha}^{A} \sigma^{\alpha}\right) d^{D-1} \sigma
\end{aligned}
$$

where $\Sigma_{M}^{\alpha} \equiv \Sigma_{\nu}^{N} \eta^{\nu \alpha} \eta_{M N}$ (and the similarly for ${ }^{1} X_{\mu}^{M}$ ).

To construct the stress term one uses the expansion of the Einstein tensor in powers of $h_{M N}$ in the harmonic gauge:

$$
G_{M N}=-\frac{\varkappa}{2} \square \psi_{M N}-\frac{\varkappa^{2}}{2} S_{M N}+\sum_{n>2} \varkappa^{n} N_{M N}^{(n)},
$$

where $\square=\partial_{M} \partial^{M}$ is the flat d'Alembert operator, $S_{M N}$ is the $\mathcal{O}\left(h^{2}\right)$ part of $G_{M N}$ given by

$$
\begin{gathered}
S_{M N}=h_{M}^{P, Q}\left(h_{N Q, P}-h_{N P, Q}\right)+h^{P Q}\left(h_{M P, N Q}+h_{N P, M Q}-h_{P Q, M N}-h_{M N, P Q}\right) \\
-\frac{1}{2} h_{, M}^{P Q} h_{P Q, N}-\frac{1}{2} h_{M N} \square h+\frac{1}{2} \eta_{M N}\left(2 h^{P Q} \square h_{P Q}-h_{P Q, L} h^{P L, Q}+\frac{3}{2} h_{P Q, L} h^{P Q, L}\right),
\end{gathered}
$$

and the last term collects higher orders. The radiation amplitude in the leading order will contain contribution of stresses arising as interaction, rather than self-action. It will be therefore convenient to consider $S_{M N}$ as a quadratic form of $h$ keeping in mind that in each term we have to retain only the mixed products of particle's and wall's contributions, so we will use the notation $S_{M N}(h, h)$ in all case when we need to state this explicitly.

\footnotetext{
${ }^{3}$ Symmetrization over two indices is defined as $A_{(M N)} \equiv\left(A_{M N}+A_{N M}\right) / 2$.
} 
This notation will be also useful to distinguish the orders of expansions used in various occasions. In particular in the source term in the eq. (4.1) for the second order metric deviation one has to use the squares of the first-order quantities ${ }^{1} h_{M N}$ in $S$ keeping only the products of $h \bar{h}$-terms and not $\bar{h} \bar{h}$ and $h h$ responsible for the self-action. Note that the $S$-part of the source is non-local contrary to the particle and the wall terms in space-time. This non-locality is due to the non-linearity of the underlying full Einstein theory and, as will be shown, it leads to an important difference in the radiation spectrum compared with the results of linear theories like electromagnetism. More detailed discussions of this point can be found in [63] within the four-dimensional theory, and in [64] in arbitrary dimensions within a simpler scalar model.

Using the equations for the first-order fields it is straightforward to verify that

$$
\partial_{N} \tau^{M N}=0
$$

which guarantees the validity of the gauge fixing condition (2.8) to this order.

\subsection{New derivation}

One starts with the particle equation of motion in an external gravitational field (3.3) written in terms of the covectors:

$$
\ddot{z}_{M}=\frac{1}{2} g_{A B, M} \dot{z}^{A} \dot{z}^{B} .
$$

The idea is to present the change of the particle momentum $\Delta \bar{P}_{M}$ (eventually we will consider the full time of motion) as the integral over the entire space-time. This is done passing to the energy-momentum tensor:

$$
\Delta \bar{P}_{M}=-m \int \ddot{z}_{M} d s=-\frac{1}{2} \int g_{A B, M} \bar{T}^{A B} \sqrt{-g} d^{D} x .
$$

Similarly we can present the change of the momentum of the wall, this quantity is assumed to be finite, while the momentum itself is infinite (for more detailed discussion see [34]). Using the eq. (2.2) we obtain:

$$
\begin{aligned}
\Delta P_{M} & =-\mu \int \partial_{\mu}\left(X_{\nu}^{N} g_{M N} \gamma^{\mu \nu} \sqrt{-\gamma}\right) d^{D-1} \sigma \\
& =-\frac{\mu}{2} \int g_{A B, M} \Sigma^{A B} \sqrt{-\gamma} d^{D-1} \sigma \\
& =-\frac{1}{2} \int g_{A B, M} T^{A B} \sqrt{-g} d^{D} x .
\end{aligned}
$$

Denote the total matter energy-momentum tensor $\mathrm{T}^{M N} \equiv \bar{T}^{M N}+T^{M N}$. Since $\mathrm{T}^{M N}$ is a symmetric conserved tensor, one has

$$
\mathrm{T}_{M ; A}^{A}=\frac{1}{\sqrt{-g}}\left(\mathrm{~T}_{M}^{A} \sqrt{-g}\right)_{, A}-\frac{1}{2} g_{A B, M} \mathrm{~T}^{A B}=0 .
$$

The change of the total momentum $\Delta \mathrm{P}_{M}$ will read:

$$
\Delta \mathrm{P}_{M}=-\int\left(\mathrm{T}_{M}^{A} \sqrt{-g}\right)_{, A} d^{D} x=-\int\left(\mathrm{T}_{M, A}^{A} \sqrt{-g}+\mathrm{T}_{M}^{A} \sqrt{-g}, A\right) d^{D} x
$$


or, equivalently,

$$
\Delta \mathrm{P}_{M}=-\int\left[\mathrm{T}_{M, A}^{A}+\frac{1}{2} \mathrm{~T}_{M}^{A} g^{N L} g_{N L, A}\right] \sqrt{-g} d^{D} x .
$$

Now we use the Einstein equations $\mathrm{T}_{M}^{A}=2 G_{M}^{A} / \varkappa^{2}$ and expand the Einstein tensor in order to express the quantities in terms of metric deviations of various orders. Since the expected radiation power is quadratic in the amplitude which itself is the second order quantity, we will need here to keep terms up to fourth order. Denoting the third order tensor in (4.5) as $N_{M N}^{(3)} \equiv C_{M N}$ and the quatric term as $N_{M N}^{(4)} \equiv Q_{M N}$, we will have:

$$
\begin{aligned}
G_{M N}= & -\frac{\varkappa}{2} \square{ }^{1} \psi_{M N}+\left[-\frac{\varkappa}{2} \square\left({ }^{2} \psi_{M N}\right)-\frac{\varkappa^{2}}{2} S_{M N}\left({ }^{1} h,{ }^{1} h\right)\right] \\
+ & {\left[-\frac{\varkappa}{2} \square\left({ }^{3} \psi_{M N}\right)-\varkappa^{2} S_{M N}\left({ }^{2} h,{ }^{1} h\right)+\varkappa^{3} C_{M N}\left({ }^{1} h,{ }^{1} h,{ }^{1} h\right)\right] } \\
+ & {\left[-\frac{\varkappa}{2} \square\left({ }^{4} \psi_{M N}\right)-\frac{\varkappa^{2}}{2} S_{M N}\left({ }^{2} h,{ }^{2} h\right)+3 \varkappa^{3} C_{M N}\left({ }^{2} h,{ }^{1} h,{ }^{1} h\right)\right.} \\
& \left.-\varkappa^{2} S_{M N}\left({ }^{3} h,{ }^{1} h\right)+\varkappa^{4} Q_{M N}\left({ }^{1} h,{ }^{1} h,{ }^{1} h,{ }^{1} h\right)\right],
\end{aligned}
$$

where multiple arguments in the cubic and quatric terms indicate the order of variables in the corresponding products. Since in each order the Einstein equations read:

$$
{ }^{k+1} G_{M N}=\frac{\varkappa^{2}}{2}{ }^{k} \mathrm{~T}_{M N}
$$

one obtains the following two lowest order equations:

$$
\begin{aligned}
& \square^{1} \psi_{M}^{N}=-\varkappa^{0} \mathrm{~T}_{M}^{N} \\
& \square^{2} \psi_{M}^{N}=-\varkappa\left({ }^{1} \mathrm{~T}_{M}^{N}+S_{M}^{N}\right) .
\end{aligned}
$$

The sum $\tau_{M}^{N} \equiv{ }^{1} \mathrm{~T}_{M}^{N}+S_{M}^{N}$ has zero divergence in accordance with the gauge choice in each order of $\psi_{M N}$. Recall again that in all products of the first order metric deviations one has to keep only the crossed particle-brane terms.

Replacing the stress-energy tensor by an expanded Einstein tensor (4.10) one has to eliminate all contributions of the first-order (non-radiative) $\psi_{M N}$; thus the lowest order of $g^{N L} g_{N L, A}$ is $\varkappa^{2} h_{A}$. The lowest order of $\mathrm{T}_{M, A}^{A}$ is ${ }^{1} \mathrm{~T}_{M, A}^{A}$ by virtue of ${ }^{0} \mathrm{~T}_{M, A}^{A}=-\square^{1} \psi_{M, A}^{A}=0$ by the gauge fixing. Finally, the lowest order of $\mathrm{T}_{M}^{A}$ is ${ }^{1} \mathrm{~T}_{M}^{A}$ due to (4.11), described in details in [65].

Hence:

- Instead of $\mathrm{T}_{M, A}^{A}$ in (4.9) we write $2 G_{M, A}^{A} / \varkappa^{2}$, then substitute all tower (4.10), eliminate all $\square\left({ }^{k+1} \psi_{M, A}^{A}\right)=0$ and all terms with ${ }^{1} h$ as multiplier, ${ }^{4}$ therefore

$$
\mathrm{T}_{M, A}^{A} \simeq-S_{M, A}^{A}\left({ }^{2} h,{ }^{2} h\right) \quad \text { plus higher orders; }
$$

- The same procedure applied to $\mathrm{T}_{M}^{A} g^{N L} g_{N L, A}$, gives

\footnotetext{
${ }^{4}$ Such neglects will be denoted as $\simeq$.
}

$$
\frac{1}{2} \mathrm{~T}_{M}^{A} g^{N L} g_{N L, A} \simeq-\frac{1}{2}{ }^{2} h_{, A} \square^{2} \psi_{M}^{A} \quad \text { plus higher orders. }
$$


Substituting this into (4.9) one gets

$$
\Delta P_{M}=\int\left[S_{M, A}^{A}\left({ }^{2} h,{ }^{2} h\right)+\frac{1}{2}^{2} h{ }_{, A} \square^{2} \psi_{M}^{A}\right] \sqrt{-g} d^{D} x
$$

where $S_{M}^{N}$ with mixed indices is defined in the same way and reads

$$
S_{M}^{N}=-\frac{1}{\varkappa^{2}}\left(2 G_{M}^{(2) N}+\varkappa \square \psi_{M}^{N}\right) .
$$

Eventually (also omitting all ${ }^{1} h$-terms) ${ }^{5}$

$$
S_{M}^{N}\left({ }^{2} h,{ }^{2} h\right)=\eta^{N L} S_{M L}\left({ }^{2} h,{ }^{2} h\right)-{ }^{2} h^{N L} \square^{2} \psi_{M L} .
$$

Calculating the divergences

$$
\partial^{N} S_{M N}\left({ }^{2} h,{ }^{2} h\right)=\frac{1}{2} \square^{2} \psi^{A B}{ }^{2} h_{A B, M}-\frac{1}{2}{ }^{2} h_{M N} \square^{2} h^{, N}+{ }^{2} h^{A B} \square^{2} h_{M A, B}
$$

and

$$
\partial_{N}\left({ }^{2} h^{N L} \square^{2} \psi_{M L}\right)=\frac{1}{2}{ }^{2} h^{, L} \square^{2} \psi_{M L}+{ }^{2} h^{N L} \square^{2} h_{M L, N}-\frac{1}{2}{ }^{2} h_{M N} \square^{2} h^{, N}
$$

and substituting into the eq. (4.15), to arrive at

$$
\Delta P_{M}=\frac{1}{2} \int{ }^{2} h_{A B, M} \square^{2} \psi^{A B}=-\frac{\varkappa}{2} \int{ }^{2} h_{A B, M} \tau^{A B} d^{D} x .
$$

This is a standard representation of the energy-momentum loss as radiation reaction work, see, e.g., [65]. Then passing to the momentum representation and taking $M=0$ one ends up with the following expression for the emitted energy

$$
E_{\mathrm{rad}}=\frac{\varkappa^{2}}{4(2 \pi)^{D-1}} \sum_{\mathcal{P}} \int_{0}^{\infty} \omega^{D-2} d \omega \int_{S^{D-2}} d \Omega\left|\varepsilon_{\mathcal{P}}^{S N} \tau_{S N}(k)\right|^{2},
$$

where $\left\{\varepsilon_{\mathcal{P}}\right\}$ represents the set of $D(D-3) / 2$ polarization tensors, to be constructed below.

To summarize, we have reproduced the familiar formula for gravitational radiation without appealing to asymptotic behavior, which is far from being trivial in our problem (see more detailed discussion in [33]). This does not mean that these conditions are irrelevant: they still can manifest themselves in spoiling the convergence properties of the integral. This is what will actually happen, but the use of the integrand as the spectralangular distribution of the radiation energy still makes sense.

\subsection{Polarization tensors}

Here we construct the polarization states in the gauge convenient to further calculations. In $D$ dimensions there are $D(D-3) / 2$ independent second-rank symmetric tensors $\varepsilon^{\mathcal{P}}$, satisfying the following conditions:

- transversality:

$$
k^{N} \varepsilon_{M N}^{\mathcal{P}}=0, \quad \bar{k}^{N} \varepsilon_{M N}^{\mathcal{P}}=0,
$$

where $k^{M}=(\omega, \mathbf{k}), \bar{k}^{M}=(\omega,-\mathbf{k}) ;$

\footnotetext{
${ }^{5}$ Note that we started with mixed indices tensor, but in fact we compute the perturbative expansion of $G_{M N}$ with lowercase indices.
} 
- tracelessness:

$$
\eta^{M N} \varepsilon_{M N}^{\mathcal{P}}=0
$$

- orthonormality:

$$
\varepsilon_{M N}^{\mathcal{P}} \varepsilon^{M N \mathcal{P}^{\prime}}=\delta^{\mathcal{P} \mathcal{P}^{\prime}}
$$

To proceed, we first construct $D-2$ space-like unit vectors $e_{a}^{M}$ orthogonal to $k$ and $\bar{k}$ and ${ }^{6}$ among themselves:

$$
\eta_{M N} e_{a}^{M} e_{b}^{N}=-\delta_{a b}, \quad k_{M} e_{a}^{M}=0, \quad \bar{k}_{M} e_{a}^{M}=0 .
$$

To specify them further, introduce the unit space-like vector $\underline{n}\left(\underline{n}^{2}=-1\right)$, associated with the unit sphere $S^{D-3}$ within the DW, and the angle $\psi$ between $\mathbf{k}$ and the $z$-axis (the line of particle motion). Then the graviton wave-vector will be parametrized as

$$
\mathbf{k}=\omega(\underline{n} \sin \psi, \cos \psi) .
$$

Next parametrize the sphere $S^{D-3}$ by $(D-4)$ polar angles $\theta_{1}, \theta_{2}, \ldots, \theta_{D-3}$ running from 0 to $\pi$ and one azimuthal angle $\phi$ running from 0 to $2 \pi$. Then the desired orthogonal $D-2$ unit vectors will consist of the following two

$$
e^{\psi}=(0, \underline{n} \cos \psi,-\sin \psi), \quad e^{\phi}=\left(0, \partial_{\phi} \underline{n}, 0\right)
$$

and the $D-4$ vectors

$$
e^{i}=\left(0, \partial_{\theta_{i}} \underline{n}, 0\right) .
$$

They all satisfy the conditions (4.19) and have no temporal component by construction. From them only the vector $e^{\psi}$ has the bulk component, while the remaining vectors belong entirely to the wall and satisfy the mutual orthogonality conditions:

$$
\eta_{\mu \nu} e_{a}^{\mu} e_{b}^{\nu}=-\delta_{a b}, \quad \delta_{i j} e_{a}^{i} e_{b}^{j}=\delta_{a b}, \quad a, b=\phi, \theta_{i} .
$$

Now, using the above unit vectors, define the following two sets of symmetric polarization tensors:

- $(D-2)(D-3) / 2$ tensors labeled by pairwise indices $r s$ :

$$
\varepsilon_{M N}^{r s}=\frac{e_{M}^{r} e_{N}^{s}+e_{M}^{s} e_{N}^{r}}{\sqrt{2}} \quad r, s=\phi, i, \psi,
$$

- $D-3$ tensors labeled by a single index $\alpha=i, z$

$$
\varepsilon_{M N}^{\alpha}=\frac{1}{\sqrt{\alpha(\alpha+1)}}\left[\alpha e_{M}^{\alpha} e_{N}^{\alpha}-\sum_{\beta=1}^{\alpha-1} e_{M}^{\beta} e_{N}^{\beta}-e_{M}^{\phi} e_{N}^{\phi}\right],
$$

\footnotetext{
${ }^{6}$ This also implies orthogonality to the time direction vector $(k+\bar{k}) / 2 \omega=(1,0, \ldots, 0)$.
} 
where the last one can be rewritten as

$$
\varepsilon_{M N}^{z}=\frac{(D-3) e_{M}^{\psi} e_{N}^{\psi}-\sum_{j=1}^{D-4} e_{M}^{j} e_{N}^{j}-e_{M}^{\phi} e_{N}^{\phi}}{\sqrt{(D-3)(D-2)}}
$$

It is the only one possessing the bulk indices via the $e_{M}^{\psi} e_{N}^{\psi}$-term.

For future purposes, it is worth noting that the polarization tensors $\varepsilon^{i \psi}, \varepsilon^{\phi \psi}$ and $\varepsilon^{z}$, with $i=\phi, \theta_{i}$ contain bulk indices ( $z$-directed) through the vector $e_{M}^{\psi}$, while the remaining $\varepsilon^{i j}$, and $\varepsilon^{i}$ do not.

\section{Radiation amplitudes}

According to eq. (4.17) we have to compute the polarization projections of the effective tensor current on the graviton mass-shell, $k^{2}=0$. The total current $\tau^{\mu \nu}(4.2)$ consists of three terms: the brane term ${ }^{1} T_{M N}(k)$, comprising contribution of the reversible deformation due to the variable gravitational field of the moving particle and the shock branon wave arising at the moment of piercing; the particle term ${ }^{1} \bar{T}_{M N}(k)$, encompassing corrections to the free motion due to the gravitational field of the brane; and $S_{M N}(k)$, the bilinear product of $h_{M N}$ and $\bar{h}_{M N}$, which is the stress-tensor of the gravitational field. It is worth noting that though the sum of the three terms is uniquely defined up to longitudinal $k^{M}$ terms vanishing under polarization projection, each separate contribution is gauge dependent, so the following calculation of separate terms is associated with specific gauge choices for the brane and the particle which were described above and will be commented again later.

\subsection{The brane amplitude}

The first-order brane stress-tensor in the coordinate representation is given by (4.4). Specifying the variations of the world-volume embedding functions as ${ }^{1} X^{N}=\Phi(\sigma) \delta_{z}^{N}$ and passing to the Fourier-transform we obtain:

$$
\begin{aligned}
{ }^{1} T_{M N}(k)=\frac{\mu}{4 \pi} \int[ & -4 i k_{\lambda} \Phi(q) \Sigma_{(M}^{\lambda} \eta_{N) z}+4 \Sigma_{(M}^{\lambda} \bar{h}_{N) \lambda}(q)-2 \Sigma_{M}^{\mu} \Sigma_{N}^{\nu} \bar{h}_{\mu \nu}(q) \\
& \left.+\Xi_{M N}\left(\bar{h}_{z z}(q)-2 i k^{z} \Phi(q)\right)\right] \delta^{D-1}\left(k^{\mu}-q^{\mu}\right) d^{D} q
\end{aligned}
$$

where $\Phi\left(q^{M}\right)$ is defined by the eqs. (3.18), (3.19) and (3.20) and reads

$$
\Phi\left(q^{M}\right)=-\frac{i \pi \varkappa^{2} m}{\gamma} \frac{q^{z} \delta\left(q^{0}-v q^{z}\right)}{q_{M} q^{M}\left(q_{\mu} q^{\mu}+2 i \epsilon q^{0}\right)}\left(\gamma^{2} v^{2}+\frac{1}{D-2}\right) .
$$

Now compute contractions of the various terms in (5.1) with the polarization tensors. Taking into account that $\bar{h}_{M N}\left(q^{M}\right)$ in (2.11) consists of $u_{M} u_{N}$ and $\eta_{M N}$ terms, one can establish the rules:

- the products of $\delta_{(M}^{\lambda} \eta_{N) z}$ with $\varepsilon_{i j}^{M N}$ and $\varepsilon_{i}^{M N}$ vanish since $e_{i}$ have no $z$-component; 
- the combination $k_{\lambda} \delta_{(M}^{\lambda} \eta_{N) z}$ has zero contractions with the polarization tensors $\varepsilon_{i \psi}^{M N}$ by virtue of orthogonality of $k^{M}$ and $e_{i}^{M}$;

- the contractions of $\Sigma_{M}^{\mu} \Sigma_{N}^{\nu} u_{\mu} u_{\nu}$ with all polarization tensors are zero since $u_{\mu}$ has only temporal non-vanishing component, while the corresponding components of $e_{i}^{M}$ and $e_{\psi}^{M}$ are zero;

- the contraction $\Sigma_{M}^{\mu} \Sigma_{N}^{\nu} \eta_{\mu \nu} \varepsilon_{i j}^{M N}$ vanishes by virtue of the mutual orthogonality of $e_{i}$ and $e_{j}$ in the brane spatial sector;

- the term $\Sigma_{M}^{\mu} \Sigma_{N}^{\nu} \eta_{\mu \nu} \varepsilon_{i}^{M N}$ is zero due to tracelessness in the brane spatial sector;

- the term $\Xi_{M N} \varepsilon_{i \psi}^{M N}$ vanishes since the vector $\underline{n}$ is unit and hence $\underline{n} \cdot \partial_{\theta_{i}} \underline{n}=0$;

- finally, the products of $u_{\lambda} u_{(M} \Sigma_{N)}^{\lambda}$ from $h_{\lambda\left(M{ }^{2}\right)}$ with polarizations $\varepsilon_{i j}^{M N}, \varepsilon_{i \psi}^{M N}$ and $\varepsilon_{i}^{M N}$ vanish since the vectors entering them do not have both $t$ - and $z$-components.

Thus we see that the only non-zero polarization projection comes from $\varepsilon_{z}$, so one is left with the only scalar amplitude $T_{z}(k) \equiv T_{M N}(k) \varepsilon_{z}^{M N}$. Moreover, similar considerations imply that one can truncate the relevant polarization tensor to

$$
\varepsilon_{z}^{M N}=\sqrt{\frac{D-3}{D-2}} e_{\psi}^{M} e_{\psi}^{N} .
$$

The non-vanishing contractions are:

$$
\Sigma_{M}^{\mu} \Sigma_{N}^{\nu} \eta_{\mu \nu} \varepsilon_{z}^{M N}=\sqrt{\frac{D-3}{D-2}} \sin ^{2} \psi, \quad k_{\lambda} \delta_{(M}^{\lambda} \eta_{N) z} \varepsilon_{z}^{M N}=-\sqrt{\frac{D-3}{D-2}} \omega \cos \psi \sin ^{2} \psi .
$$

So collecting all the non-zero terms and integrating over $q$ we obtain:

$$
{ }^{1} T_{z}(k)=-\sqrt{\frac{D-3}{D-2}} \frac{\varkappa^{2} \mu m}{2} \frac{\gamma v \sin ^{2} \psi}{\omega^{2}+k_{\perp}^{2} \gamma^{2} v^{2}}\left[\frac{\omega^{2} \cos \psi}{v\left(k_{\mu} k^{\mu}+2 i \epsilon k^{0}\right)}\left(\gamma^{2} v^{2}+\alpha_{D}\right)+\gamma^{2} v^{2}-\alpha_{D}\right],
$$

where $k_{\perp}^{2} \equiv \delta_{i j} k^{i} k^{j}$ and $\alpha_{D} \equiv(D-2)^{-1}$. Taking into account the on-shell condition $k_{M} k^{M}=0$, this quantity can be rewritten as

$$
\begin{aligned}
{ }^{1} T_{z}(k)= & -\sqrt{\frac{D-3}{D-2}} \frac{\varkappa^{2} \mu m}{2 \omega^{2}} \frac{\gamma v \sin ^{2} \psi}{1+\gamma^{2} v^{2} \sin ^{2} \psi} \\
& \times\left[\frac{\cos \psi}{v\left[\cos ^{2} \psi+2 i \epsilon k^{0}\right]}\left(\gamma^{2} v^{2}+\frac{1}{D-2}\right)+\gamma^{2} v^{2}-\frac{1}{D-2}\right] .
\end{aligned}
$$

We note the infrared divergence of this amplitude at $\omega \rightarrow 0$, which is not surprising since our procedure did not take into account the finite depth of the piercing layer. We will deal with this problem later on. Another interesting feature is that the amplitude remains non-zero in the limit $v \rightarrow 0$. This is related to branon excitation which takes place even with infinitesimal $v$, for more details see [31]. Also, one can see that the amplitude diverges as $\psi \rightarrow \pi / 2$, i.e. along the DW. This divergence is another artefact of our approximation, to be dealt with later on. 
The divergence of the brane amplitude along the wall is due to brane excitation $\Phi$. If one puts $\Phi=0$ by hand (this may correspond to $\mathbb{Z}_{2}$-symmetric braneworld models or to the case of two mirror particles impinging upon the wall), then

$$
\left.{ }^{1} T_{z}(k)\right|_{\Phi=0}=-\sqrt{\frac{D-3}{D-2}} \frac{\varkappa^{2} \mu \mathcal{E}}{2 \omega^{2}} \frac{v \sin ^{2} \psi}{1+\gamma^{2} v^{2} \sin ^{2} \psi}\left[\gamma^{2} v^{2}-\frac{1}{D-2}\right] .
$$

Thereby in this case the amplitude does not blow up at $\psi=\pi / 2$ and the angular distribution is finite.

\subsection{The particle amplitude}

The first-order particle stress-tensor was found in the preceding section in the coordinate representation (4.3):

$$
{ }^{1} \bar{T}_{M N}=\frac{m}{2} \int\left(4^{1} \dot{z}_{(M} u_{N)}+\varkappa\left(4 u^{P} h_{P(M} u_{N)}-h u_{M} u_{N}\right)-2 u_{M} u_{N}\left({ }^{1} z \cdot \nabla\right)\right) \delta^{D}(x-u \tau) d \tau
$$

with $z^{M}$ given by (3.2) and (3.5). The corresponding amplitude in the momentum representation reads:

$$
\begin{aligned}
{ }^{1} \bar{T}_{M N}(k)=\frac{m}{2} \int d \tau e^{i(k u) \tau}[ & {\left[2 i\left(k^{1} z\right) u_{M} u_{N}+4 u_{(M}{ }^{1} \dot{z}_{N)}\right.} \\
& \left.-\varkappa h(\tau) u_{M} u_{N}+4 \varkappa u^{P} u_{(M} h_{N) P}(\tau)\right],
\end{aligned}
$$

where the brane gravitational field $h_{M N}(\tau)$ is given by (2.14), restricted to the unperturbed trajectory:

$$
h_{M N}(\tau)=\frac{\varkappa \mu}{2}\left(\Xi_{M N}-\frac{D-1}{D-2} \eta_{M N}\right) \gamma v|\tau|,
$$

and ${ }^{1} z^{M}(\tau)$ is given by (3.5). For ${ }^{1} \bar{T}_{M N}(k)$ the non-zero contribution gives only the polarization $\varepsilon_{z}$, yielding the product ${ }^{1} \bar{T}_{z}(k)$. Contracting (5.7) with (5.3) and using the integrals

$$
\int_{-\infty}^{\infty} \mathrm{e}^{i \alpha \tau}|\tau| d \tau=-\frac{2}{\alpha^{2}}, \quad \int_{-\infty}^{\infty} \mathrm{e}^{i \alpha \tau} \tau^{2} \operatorname{sgn}(\tau) d \tau=-\frac{4 i}{\alpha^{3}}
$$

one obtains

$$
{ }^{1} \bar{T}_{z}(k)=-\left[\frac{D-3}{4(D-2)^{3}}\right]^{1 / 2} \frac{\varkappa^{2} \mu m v}{\gamma \omega^{2}} \frac{\left[(D-2) \gamma^{2} v^{2}-1\right] v \cos \psi+2}{(1-v \cos \psi)^{3}} \sin ^{2} \psi .
$$

This amplitude, apart from the infrared, has also the angular divergence at $\psi=0$ in the case of the massless particle $v=1$. This is the well-known collinear divergence encountered in quantum perturbation theory for interacting massless particles. In classical theory this is the line divergence of the retarded potentials [66]. 


\subsection{The stress contribution}

The stress tensor is given by eq. (4.6). Substituting $h_{M N}=h_{M N}+\bar{h}_{M N}$ and keeping only the cross terms one obtains a bi-linear form of $h$ and $\bar{h}$. Anticipating zero contractions of $\eta_{M N}$-part with traceless polarization tensors, one can drop it from the beginning. The Fourier transform of the product of two fields becomes convolution in the momentum representation presented by the integrals over the variable $q^{M}$. The following integrals are useful in such a computation:

$$
\begin{aligned}
& \int \frac{\delta(q u) \delta^{D-1}\left(q^{\mu}\right)}{q^{2}(k-q)^{2}} d^{D} q=\frac{\gamma^{3} v^{3}}{(k u)^{3}(\bar{k} u)} \\
& \int \frac{\delta(q u) \delta^{D-1}\left(q^{\mu}\right)}{q^{2}(k-q)^{2}} q_{M_{1}} \ldots q_{M_{n}} d^{D} q=\left(\frac{k u}{\gamma v}\right)^{n-3} \frac{\delta_{M_{1} z} \ldots \delta_{M_{n} z}}{(\bar{k} u)}
\end{aligned}
$$

(with $\bar{k}^{M}$ introduced in (4.18)). After lengthy but straightforward calculations one obtains the following expression:

$$
\begin{gathered}
S_{M N}(k)=\frac{\varkappa^{2} \mu m}{\gamma v \tilde{q}^{2}(k-\tilde{q})^{2}}\left[\Xi_{P Q} k^{P} k^{Q} u_{M} u_{N}+\left(\gamma^{2} v^{2}-\frac{1}{D-2}\right) \tilde{q}_{M} \tilde{q}_{N}+(k u)^{2} \Xi_{M N}+\right. \\
\left.+[(k u)+(\bar{k} u)] \tilde{q}_{(M} u_{N)}-2(k u) k^{A} \Xi_{A(M} u_{N)}\right],
\end{gathered}
$$

with $\tilde{q}^{M}=\left(0, \ldots, 0, k^{z}-k^{0} / v\right)$. Here the longitudinal terms proportional to $k_{(M} B_{N)}$ (with any $D$-vector $B^{M}$ ) were also omitted in view of transversality of polarization tensors. On shell $k^{2}=0$ this expression reduces to

$$
\begin{gathered}
S_{M N}(k)=\frac{\varkappa^{2} \mu m \gamma^{3} v^{3}}{(k u)^{3}(\bar{k} u)}\left[\left(k^{z}\right)^{2} u_{M} u_{N}+\left(\gamma^{2} v^{2}-\frac{1}{D-2}\right) \tilde{q}_{M} \tilde{q}_{N}+(k u)^{2} \Xi_{M N}\right. \\
\left.+2 \gamma k^{0} \tilde{q}_{(M} u_{N)}-2(k u) k^{A} \Xi_{A(M} u_{N)}\right] .
\end{gathered}
$$

One can notice that the stress tensor contains the same tensor structures as ${ }^{1} \bar{T}_{M N}(k)$ and ${ }^{1} T_{M N}(k)$, so the only polarization tensor giving non-zero result will be again $\varepsilon_{z}^{M N}$. The following contractions can be easily found

$$
\tilde{q}_{M} e_{\psi}^{M}=-\frac{(k u)}{\gamma v} \sin \psi, \quad k^{A} \Xi_{A(M} u_{N)} \varepsilon_{z}^{M N}=-\sqrt{\frac{D-3}{D-2}} \gamma v \omega \sin ^{2} \psi \cos \psi,
$$

in addition to (5.4). Using them, the projected stress-tensor amplitude can be presented as:

$$
S_{z}(k)=\sqrt{\frac{D-3}{D-2}} \frac{\varkappa^{2} \mu m v^{3} \sin ^{2} \psi}{\gamma\left(k^{0}-v k^{z}\right)^{3}\left(k^{0}+v k^{z}\right)}\left[\gamma^{2} v^{2}\left(k^{z}\right)^{2}-\frac{(k u)^{2}}{(D-2) \gamma^{2} v^{2}}\right] .
$$

Finally, using the on shell parametrization $k=\omega(1, \sin \psi \underline{n}, \cos \psi)$, one obtains:

$$
S_{z}(k)=\sqrt{\frac{D-3}{D-2}} \frac{\varkappa^{2} \mu m v}{\gamma \omega^{2}} \frac{\sin ^{2} \psi}{(1-v \cos \psi)^{3}(1+v \cos \psi)}\left[\gamma^{2} v^{4} \cos ^{2} \psi-\frac{(1-v \cos \psi)^{2}}{D-2}\right] .
$$

Here one also observes both the infrared and the angular divergences. 


\subsection{The destructive interference in the ultrarelativistic limit}

In the ultrarelativistic limit $v \rightarrow 1(\gamma \rightarrow \infty)$ both the particle and the stress amplitudes have similar behavior near the forward direction $\psi \ll 1$ which could give the leading contribution to radiation (this follows from the integrals (A.1)). However, keeping the common singular factors and expanding the rest as

$$
\sin \psi \approx \psi, \quad 1-v \cos \psi \approx \frac{\psi^{2}+\gamma^{-2}}{2}
$$

one finds for large $\gamma \gg 1$

$$
{ }^{1} \bar{T}_{z}(k)=-\frac{1}{2} \sqrt{\frac{D-3}{D-2}} \frac{\varkappa^{2} \mu \mathcal{E} \sin ^{2} \psi}{\omega^{2}(1-v \cos \psi)^{3}}\left(1+\mathcal{O}\left(\gamma^{-2}\right)\right)
$$

and

$$
S_{z}(k)=\frac{1}{2} \sqrt{\frac{D-3}{D-2}} \frac{\varkappa^{2} \mu \mathcal{E} \sin ^{2} \psi}{\omega^{2}(1-v \cos \psi)^{3}}\left(1+\mathcal{O}\left(\gamma^{-2}\right)\right)
$$

where $\mathcal{E}=m \gamma$ is the particle energy. So in the leading in $\gamma$ order, these two amplitudes exactly cancel. This is manifestation of the destructive interference which reflects the equivalence principle in the language of flat space, which was encountered in the bremsstrahlung problem for point particles [63-65, 67,68]. After cancelation of the leading terms, the sum of (5.8) and (5.9) has two orders of gamma less than the each term separately.

On the other hand, the brane amplitude (5.5) in the forward direction is approximated as

$$
\left.{ }^{1} T_{z}(k)\right|_{\psi \ll 1} \approx-\sqrt{\frac{D-3}{D-2}} \frac{\varkappa^{2} \mu \mathcal{E}}{\omega^{2}} \frac{\gamma^{2} \sin ^{2} \psi}{1+\gamma^{2} \sin ^{2} \psi}
$$

and thereby is of order of $\mathcal{O}\left(\omega^{-2} \varkappa^{2} \mu \mathcal{E}\right)$. Comparing it with $(1-v \cos \psi)^{-2}=\mathcal{O}\left(\gamma^{4}\right)$ one concludes that at $\psi \approx 0$ the brane contribution is always subleading.

Thus in the small-angle region the main contribution still comes from the sum of ${ }^{1} \bar{T}\left(k^{M}\right)$ and $S\left(k^{M}\right)$. Expanding these with more accuracy and keeping the subleading terms, one finds to the main order:

$$
\left.\tau_{z}(k)\right|_{\psi \ll 1} \approx-\frac{1}{8} \sqrt{\frac{D-3}{D-2}} \frac{\varkappa^{2} \mu \mathcal{E} \sin ^{2} \psi}{\gamma^{2} \omega^{2}(1-v \cos \psi)^{3}}\left[\frac{D+2}{D-2}+\gamma^{2} \sin ^{2} \psi\right] .
$$

The total amplitude is peaked at $\psi \sim 1 / \gamma$ in any dimensions, ${ }^{7}$ with the magnitude $\propto \gamma^{2}$. The qualitative picture in $D=4,5,6$ is shown on the figure 1 .

The dependence of the radiation amplitude on the particle Lorentz factor in $D=4$ is shown on the figure 2 .

\footnotetext{
${ }^{7}$ More precisely, the position of maximum is $\psi=\left(\frac{\sqrt{D^{2}+12}-4}{D-2}\right)^{1 / 2} / \gamma$, plus small corrections.
} 


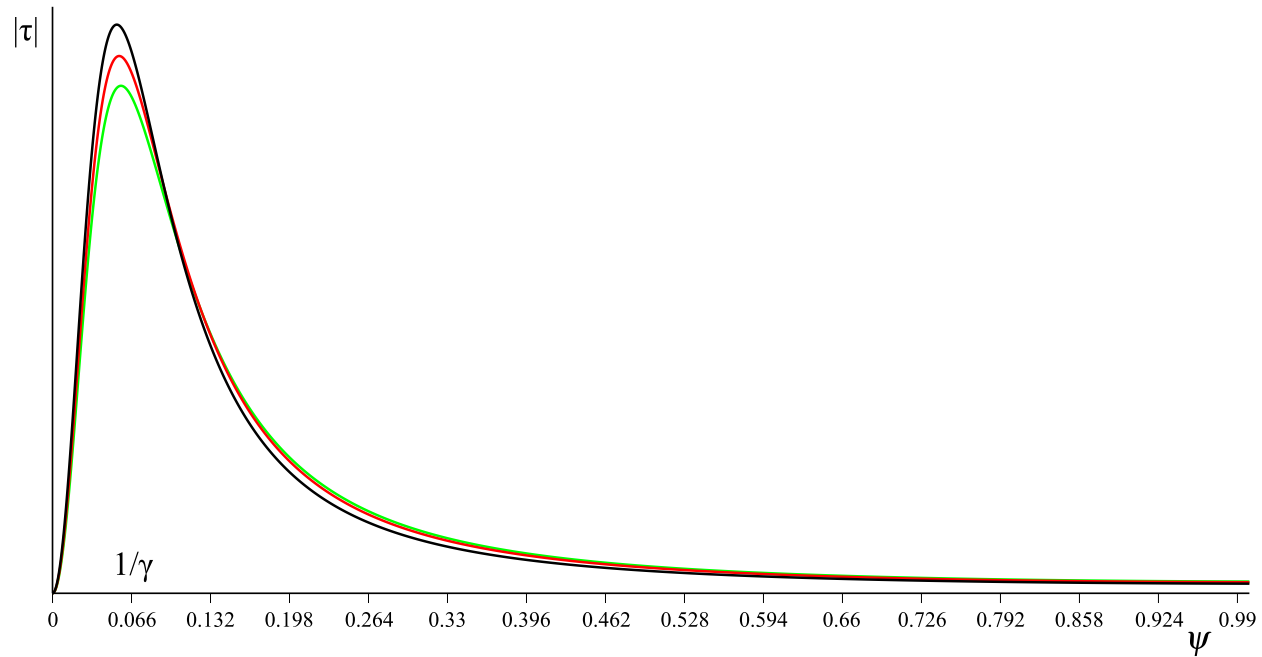

Figure 1. The angular dependence of the radiation amplitude for $\gamma=15$ in four (black), five (red) and six (green) spacetime dimensions (units $\varkappa^{2} \mu \mathcal{E} \omega^{-2}=1$ are understood).

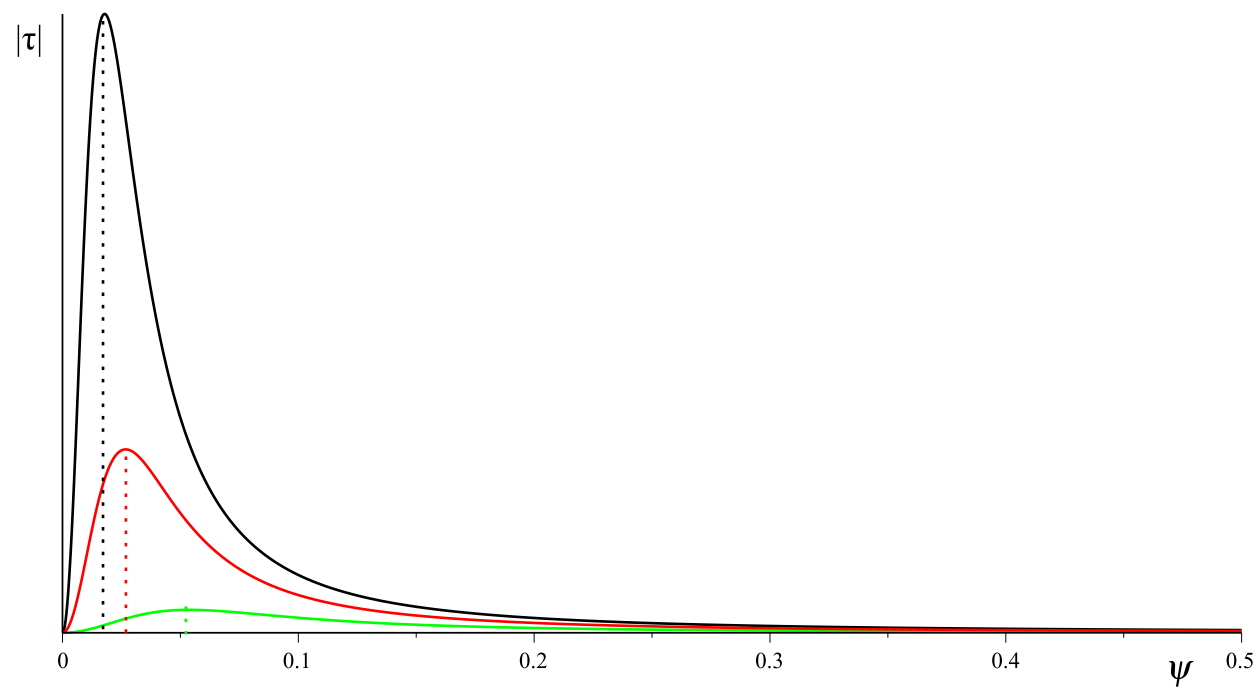

Figure 2. The angular dependence of the radiation amplitude in four dimensions for different values of Lorentz factor: $\gamma=15$ (green), $\gamma=30$ (red) and $\gamma=45$ (black) (units $\varkappa^{2} \mu \mathcal{E} \omega^{-2}=1$ are understood).

\subsection{The massless case}

The limiting case, corresponding to the piercing of DW by photons, is the case where mass tends to zero with fixed energy. The latter represents the photon's frequency in $\hbar=1$ units. The corresponding change should be observable in the angular dependence of the radiation amplitudes. Indeed, the position of the maximum $\mathcal{O}(1 / \gamma)$ for the ultrarelativistic particle goes to zero, while the height $\gamma^{2}$ blows up to infinity. 


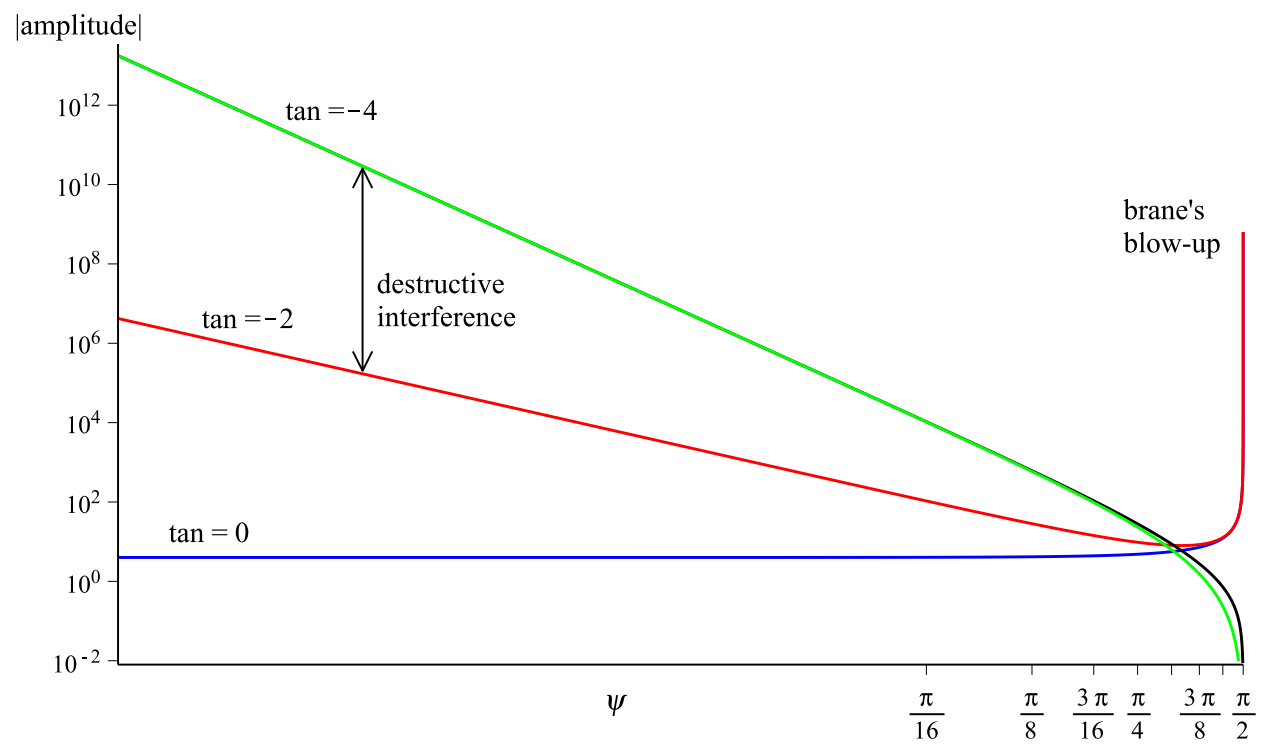

Figure 3. Angular dependence of the (absolute values) radiation amplitudes for photon: particle (black), brane (blue) and stress (green) contributions and the total amplitude (red) in doubly logarithmic mode (in units $C=1$ ).

Directly taking the limits of (5.5), (5.8) and (5.9) one gets

$$
\begin{aligned}
{ }^{1} T_{z}(k) & =-2 C\left[\frac{\cos \psi}{\cos ^{2} \psi+2 i \epsilon k^{0}}+1\right], & { }^{1} \bar{T}_{z}(k) & =-C \frac{\cos \psi \cos ^{2}(\psi / 2)}{\sin ^{4}(\psi / 2)} \\
S_{z}(k) & =C \frac{\cos ^{2} \psi}{\sin ^{4}(\psi / 2)}, & C & \equiv \sqrt{\frac{D-3}{D-2} \frac{\varkappa^{2} \mu \mathcal{E}}{4 \omega^{2}},}
\end{aligned}
$$

with no dependence on the Lorentz factor. Here $\mathcal{E}$ stands for the photon frequency. One notices that for the small angles the brane contribution is regular, while both $\bar{T}_{z}(k)$ and $S_{z}(k)$ blow up as $\psi^{-4}$. Combining them, the total amplitude in the small-angle domain reads:

$$
\tau_{z}(k)=-C \frac{\cos \psi}{\sin ^{2}(\psi / 2)},
$$

and thus blows up as $\psi^{-2}$ when $\psi$ approaches zero. Thus the destructive interference in this case consists in the diminishing of the angular blow-up power by two powers. The plot on figure 3 illustrates these observations.

Note that these curves are the same for any $D$, all $D$-dependence is contained only in the factor $C$.

To summarize this section, we list the main results:

- the radiation amplitude consists of a single polarization, responsible for emission into the bulk;

- the amplitude has the universal infrared divergence $\omega^{-2}$; 
- in the ultra-relativistic case the radiation amplitude is peaked in the forward direction, though the leading contributions of the particle and the stresses mutually cancel due to destructive interference;

- the brane amplitude blows up along the DW and remains non-zero in the limit $v \rightarrow 0$.

\section{The spectral and angular distribution of PGR}

From eq. (4.17) one obtains simple expression for the spectral-angular distribution of the total $\varepsilon_{z}$-polarized PGR:

$$
\frac{d E_{\mathrm{rad}}}{d \omega d \Omega}=\frac{\varkappa^{2}}{4(2 \pi)^{D-1}} \omega^{D-2}\left|\tau_{z}(k)\right|^{2}
$$

In view of the spherical symmetry on brane, we integrate over sphere $S^{D-3}$ (all angles except $\psi$ ) obtaining for the total emitted energy

$$
E_{\mathrm{rad}}=\frac{\varkappa^{2}}{(4 \pi)^{D / 2} \Gamma\left(\frac{D-2}{2}\right)} \int_{\omega_{\min }}^{\omega_{\max }} d \omega \omega^{D-2} \int_{\psi_{\min }}^{\pi} d \psi \sin ^{D-3} \psi\left|\tau_{z}(\omega, \psi)\right|^{2} .
$$

The integrand typically is peaked along $\psi=0$, and for massive particles (finite $\gamma$ ) it is non-divergent there (actual integration is performed according to appendix A). But in the massless limit it is divergent, so the cut-off at $\psi_{\min }$ is required. This cut-off depends on the particular physical problem which is supposed to replace our simplified model and may be either classical, or quantum. In what follows we will discuss this in more details.

Taking into account that all radiation amplitudes scale as $\omega^{-2}$ in the entire spectrum, the substitution of $\tau_{z}(k)$ into (6.1) after integration over angles leads to the frequency distribution

$$
\frac{d E_{\mathrm{rad}}}{d \omega} \propto \varkappa^{6} \mu^{2} m^{2} \omega^{D-6}
$$

This quantity is infrared-divergent in all dimensions less than six. This also is the consequence of the oversimplified nature of our model which indicates the need of the infrared cut-off. For $D \geqslant 5$ an ultraviolet cut-off is also required. These again can be classical or quantum, especially in the case of massless particles. Recall that in the classical domain we have two intrinsic length parameters in the full non-linear theory — the inverse bulk curvature $k^{-1}$ and the gravitational radius associated with the particle energy $\mathcal{E}$, namely

$$
r_{\mathcal{E}} \sim\left(\varkappa^{2} \mathcal{E}\right)^{1 /(D-3)}
$$

In addition, in more realistic DW models one encounters other physical length parameters: the DW thickness $\delta$ and the finite longitudinal size of the DW. Finally, applicability of the perturbation theory requires the distances in the bulk direction to be restricted from above by the size of the piercing layer (3.7) which is of the order of the bulk curvature radius for the photons, and is $O\left(v^{2}\right)$ for non-relativistic velocities. 


\subsection{The ultra-relativistic case: beaming in the bulk direction}

The dominant part of the total radiation still can be beamed even if the corresponding particle and stress contribution mutually cancel. Substituting (5.14) into (6.2) one encounters the competing over the polar angle $\psi: V_{6}^{D+1}, \gamma^{2} V_{6}^{D+3}$ and $\gamma^{4} V_{6}^{D+5}$, as introduced and evaluated in the appendix A. In the leading order one obtains

$$
E_{\mathrm{rad}}=\frac{(D-3) \Gamma\left(\frac{D+6}{2}\right) \Gamma\left(\frac{6-D}{2}\right)}{30(D-2)^{2}(4 \pi)^{D / 2} \Gamma(D / 2)}\left(\varkappa^{3} \mu \mathcal{E}\right)^{2} Q_{D} \gamma^{6-D},
$$

where frequency factor $Q_{D}$ is

$$
Q_{D}= \begin{cases}1 / \omega_{\min }, & D=4 \\ \ln \frac{\omega_{\max }}{\omega_{\min }}, & D=5 \\ \omega_{\max }^{D-5} /(D-5), & D>5\end{cases}
$$

The factor $\Gamma\left(\frac{6-D}{2}\right)$ indicates that for $D \geqslant 6$ the formula (A.6) is irrelevant. Indeed, for $D=6$ the integral $V_{6}^{11}$ exhibits the non-beamed logarithmic behavior (A.8). The two remaining integrals are still "beamed" (A.6), but the power of $\gamma$ in the denominator (5.14) makes the forward-direction contribution to be of the same order as non-forward contributions which are more difficult to access analytically. For $D>6$ it is not hard to combine contributions $V_{6}^{D+1}, V_{6}^{D+3}$ and $V_{6}^{D+5}$ (choosing the appropriate case out of (A.6), (A.7) or (A.8)), but the total forward radiation is negligible due to the small phase volume of the forward-direction beaming cone $\psi \lesssim \gamma^{-1}$. Thus it is natural to consider the cases $D=4$, $D=5$ and $D \geqslant 6$ for the ultrarelativistic particle separately.

$\boldsymbol{D}=\mathbf{4}$. The direct application of (6.4) yields:

$$
E_{\mathrm{rad}}=\frac{1}{80 \pi^{2}} \frac{\left(\varkappa_{4}^{3} \mu \mathcal{E}\right)^{2} \gamma^{2}}{\omega_{\min }},
$$

where we traded the particle mass in favor of the energy $\mathcal{E}=m \gamma$. Obviously this diverges in the massless limit when $\gamma \rightarrow \infty$. This divergence is a consequence of the collinear divergence of the amplitude, which requires the angular cut-off $\psi_{\min }$ :

$$
E_{\mathrm{rad}}=\frac{1}{80 \pi^{2}} \frac{\left(\varkappa_{4}^{3} \mu \mathcal{E}\right)^{2}}{\omega_{\min }\left(\psi_{\min }\right)^{2}} .
$$

It is expected that the cut-off has quantum nature, like in the case of synchrotron radiation of massless particles [45], which is beyond the scope of our treatment. Otherwise, one can think of the factor $\left(\psi_{\min }\right)^{-1}$ as an effective maximal Lorentz factor $\gamma_{*}$.

$\boldsymbol{D}=\mathbf{5}$. This case is "softer" as containing a single power of $\gamma$ : now from (6.4) we have:

$$
E_{\mathrm{rad}}=\frac{7}{3 \cdot 2^{8} \pi^{2}}\left(\varkappa_{5}^{3} \mu \mathcal{E}\right)^{2} \gamma \ln \frac{\omega_{\max }}{\omega_{\min }} .
$$

In this case one can find reasonable cut-offs from applicability conditions of our approach. The cutoffs $\omega_{\min }$ and $\omega_{\max }$ come from the corresponding coordinate cutoffs discussed in the 
appendix B. Another restriction could come from non-infinite longitudinal size of physical DW, and one has to combine them together. This stimulates us to revisit the 1st-order deformation of the brane, given by $\Phi \equiv \Phi_{\mathrm{a}}+\Phi_{\mathrm{b}}$ [32, eqs. 5.14, 5.17], with

$$
\Phi_{\mathrm{a}}=-\frac{\varkappa_{5}^{2} \mathcal{E}}{8 \pi^{2} r} \arctan \frac{r}{\gamma v t}, \quad \Phi_{\mathrm{b}}=-\frac{\varkappa_{5}^{2} \mathcal{E}}{8 \pi r} \theta(t) \theta(r-t),
$$

where $\theta(x)$ stands for the Heaviside step-function. The absolute value of both these is maximal for small $r$, hence the maximal $z$-direction brane deformation has to be taken at $r=r_{\min }\left(\right.$ for $\left.|t|>z_{\min }\right)$, or at $r=0, t=z_{\min }$ in $\Phi_{\mathrm{a}}$, yielding

$$
|\Phi|_{\max }=\frac{\varkappa_{5}^{2} \mathcal{E}}{8 \pi^{2} r_{\min }} \simeq \frac{3 r_{\mathcal{E}}^{2}}{r_{\min }},
$$

where we take into account (B.7). Demanding for the brane deflection to be secured by the "true" minimal available-for-consideration $z$-coordinate, in order to keep the validity of the perturbation theory (what implies $|\Phi|_{\max }=z_{\min }$ ), and taking into account the correlation between $z$ and $r$ for the branon wave we conclude:

$$
r_{\min } \simeq z_{\min }=\mathcal{O}\left(r_{\mathcal{E}}\right)
$$

since these two exceed the "previous" minimal values $r_{\min }=r_{\mathcal{E}} \gamma^{-1 /(D-3)}$ and $z_{\min }=$ $=r_{\mathcal{E}} \gamma^{-(D-2) /(D-3)}$ in (B.7). Thus

$$
\omega_{\max }=\mathcal{O}\left(1 / r_{\mathcal{E}}\right),
$$

independent of the graviton direction.

Second, here we are basically consider the RSII setting where the brane is fivedimensionally infinite. Let consider for the moment the case with infinite Lorentz factor within the RSII model. Note, the zeroth-order field in our model is Minkowskian everywhere. This fact preserves the conservation laws [32, 33], and the dynamics of particle and brane is self-consistent. But if we consider times

$$
t>L \equiv z_{\max }
$$

in RSII-setting, the free particle after the shock collision moves in the true RSII-metric which is exponentially decaying. Hence the corresponding branon wave, $\Phi_{\mathrm{b}}$, propagating outward the piercing point, when reaching the value $r=L$ has to be deformed significantly. ${ }^{8}$ In other words, we have to put

$$
r_{\max }=\mathcal{O}(L), \quad k_{\min }^{r}=\mathcal{O}(1 / L) .
$$

The minimal value of frequency $\omega$ is determined by the two inverse length parameters: both longitudinal, $1 / z_{\max }$, and transverse, $1 / r_{\max }$. Noticing that the two latter coincide, we conclude

$$
\omega_{\min }=\mathcal{O}(1 / L) .
$$

\footnotetext{
${ }^{8}$ Such a non-perturbative analysis lies beyond our goals here.
} 
Substituting (6.10) and (6.12) into (6.8) one obtains: ${ }^{9}$

$$
E_{\mathrm{rad}}=\frac{7}{3 \cdot 2^{8} \pi^{2}}\left(\varkappa_{5}^{3} \mu \mathcal{E}\right)^{2} \gamma \ln \frac{L}{r_{\mathcal{E}}} .
$$

Normalizing it by the particle energy, we have:

$$
\epsilon \equiv \frac{E_{\mathrm{rad}}}{\mathcal{E}} \simeq \frac{7}{2^{5}} \frac{r_{\mathcal{E}}^{2} \gamma}{L^{2}} \ln \frac{L}{r_{\mathcal{E}}}
$$

and we can consider two cases described in [34]: the resolution is that the existence of $k_{\min }^{r}$ prevents the angle $\psi$ to approach zero:

$$
\psi_{\min } \sim \arcsin \frac{k_{\min }^{r}}{\omega_{\max }} \simeq \frac{r_{\mathcal{E}}}{L} \ll 1 .
$$

Notice, the result (6.13) is got after the $\psi$-integration from 0 to $\pi$, whereas in the relativistic case we thus have to integrate the angular distribution (6.1) from $\psi_{\min }$. Since the integrand is beamed inside the cone $0<\psi \lesssim 1 / \gamma$, the final result depends upon the relation between $1 / \gamma$ and $\psi_{\min }$. Namely, expressing the radiation efficiency (6.13) in terms of inverse minimal emission angle, it estimates as

$$
\epsilon \sim \frac{r_{\mathcal{E}}}{L} \ln \frac{L}{r_{\mathcal{E}}}
$$

or $\ln \gamma_{*} / \gamma_{*}$ in terms of effective Lorentz factor $\gamma_{*} \equiv \psi_{\min }^{-1}$. Since the function $\ln x / x$ does not exceed 1 for $x>1$, there is no efficiency catastrophe in our model. In fact, we assume $L / r_{\mathcal{E}} \simeq \gamma_{*} \gg 1$, so $\epsilon \ll 1$.

$\boldsymbol{D} \geqslant \mathbf{6}$. As was deduced above, the most of the angular contribution is taken from the angles of order $\mathcal{O}(1)$. Meanwhile, in six dimensions both the "beaming" region $\psi \lesssim \mathcal{O}\left(\gamma^{-1}\right)$ and $\psi \gtrsim \mathcal{O}\left(\gamma^{-1}\right)$ contribute on equal footing, that implies for the local values of the angulardistribution curve at $\psi \simeq \mathcal{O}\left(\gamma^{-1}\right)$ to be in $\gamma$ times greater than the same one at $\psi \simeq \mathcal{O}(1)$. The plot on figure 6 confirms this conclusion. Thus we have the "local" beaming, with the total contribution as

$$
E_{\mathrm{rad}}=\frac{6}{(4 \pi)^{5}}\left(\varkappa_{6}^{3} \mu \mathcal{E}\right)^{2}\left(\ln 2 \gamma-\frac{107}{60}\right) \omega_{\max }, \quad D=6 .
$$

For $D>6$ the $\gamma \gg 1$ limit has to be applied to the region $\psi=\mathcal{O}(1)$. Doing this, one obtains the estimate

$$
\left.\tau_{z}(k)\right|_{\psi=\mathcal{O}(1)} \sim \frac{\varkappa^{2} \mu \mathcal{E}}{\omega^{2}} f(\psi), \quad E_{\mathrm{rad}} \sim\left(\varkappa^{3} \mu \mathcal{E}\right)^{2} Q_{D}
$$

where $f(\psi)$ denotes some function of order $\mathcal{O}(1)$ with no characteristic dependence on $\gamma \gg 1$ inside.

\footnotetext{
${ }^{9}$ Despite the cutoffs are defined as approximate values of declared order, we have kept the numerical pre-factor due to the logarithm changes insignificantly with respect to the deviation of approximate $\omega_{\max }$ and $\omega_{\min }$.
} 


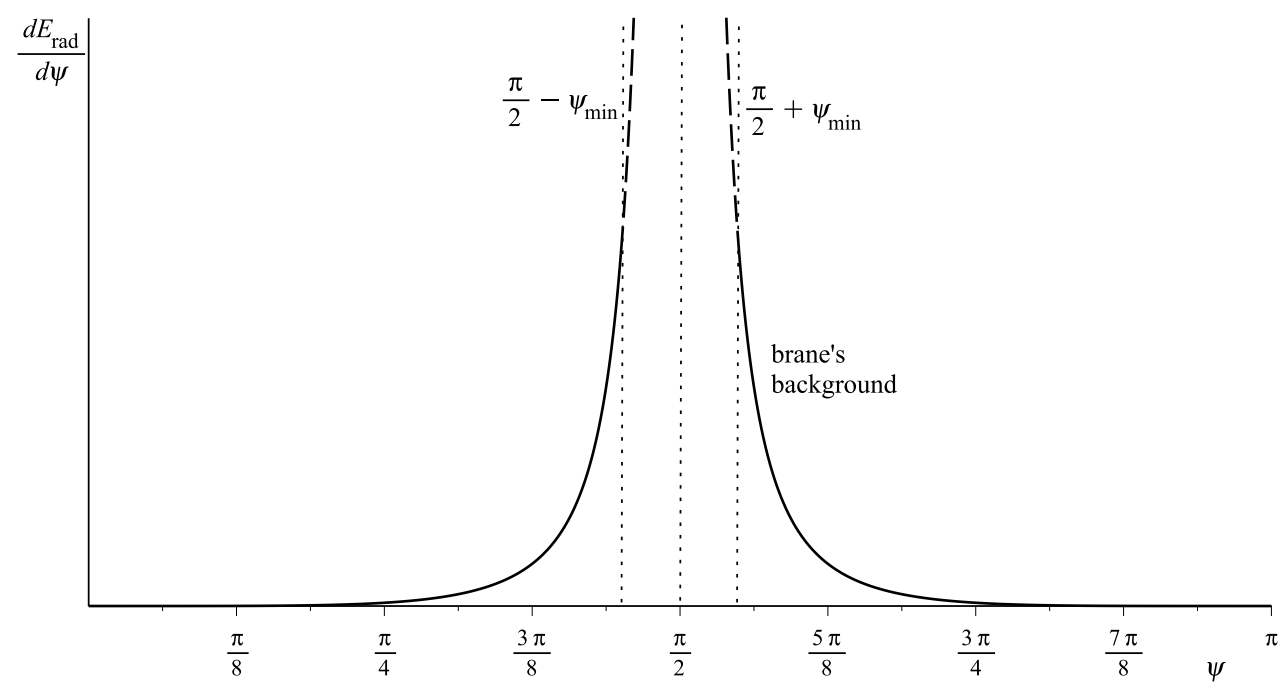

Figure 4. Angular distribution of the emitted radiation at zero-speed for $D=4$.

\subsection{Non-relativistic case: the brane contribution}

As it was mentioned above, the brane contribution survives in the limit $v \rightarrow 0$ (5.5). In this case the angular distribution is symmetric with respect to the brane's plane. Thus we deal with the pure brane configuration of waves, the corresponding plot is shown on the figure 4.

However, the term $1 / k_{z}$ in ${ }^{1} T_{z}(k)$ is presented in the generic case $v>0$, thus one can expect the blow up of the curve of the total emission angular distribution at angle $\psi$ close to $\pi / 2$, i.e. when the graviton is emitted tangent to the brane: from (5.5) we have now:

$$
\left.\tau_{z}(k)\right|_{\psi \approx \pi / 2}=-\sqrt{\frac{D-3}{D-2}} \frac{\varkappa^{2} \mu m}{2 \omega^{2} \cos \psi} \frac{\gamma}{1+\gamma^{2} v^{2}}\left(\gamma^{2} v^{2}+\frac{1}{D-2}\right) .
$$

Introducing the complement $\psi^{\prime} \equiv \pi / 2-\psi$ and estimating, we conclude:

$$
\left.\tau_{z}(k)\right|_{\psi \approx \pi / 2} \simeq-\frac{\varkappa^{2} \mu \mathcal{E}}{\omega^{2} \psi^{\prime}}
$$

This indicates to the imposing of a cutoff on the $k^{z}$ and $\psi^{\prime}$ : indeed, from the maximal $z_{\max }$ we can deduce:

$$
k_{\min }^{z}=\mathcal{O}(1 / L), \quad \psi_{\min }^{\prime}=\psi_{\min } .
$$

Integration of (6.18) yields the "brane" contribution to the spectrum:

$$
E_{\mathrm{br}} \simeq\left(\varkappa^{3} \mu \mathcal{E}\right)^{2} \frac{Q_{D+1}}{k_{\min }^{z}},
$$

with $Q_{n}$ introduced in (6.5). However, in the non-relativistic case the maximal $z$-size of applicability of our approach is $z<z_{l}(v)$ which goes to zero as $v^{2}$ for small velocities. So in the non-relativistic case one could do better considering particle motion in the exact brane background, we leave this for the future work. 


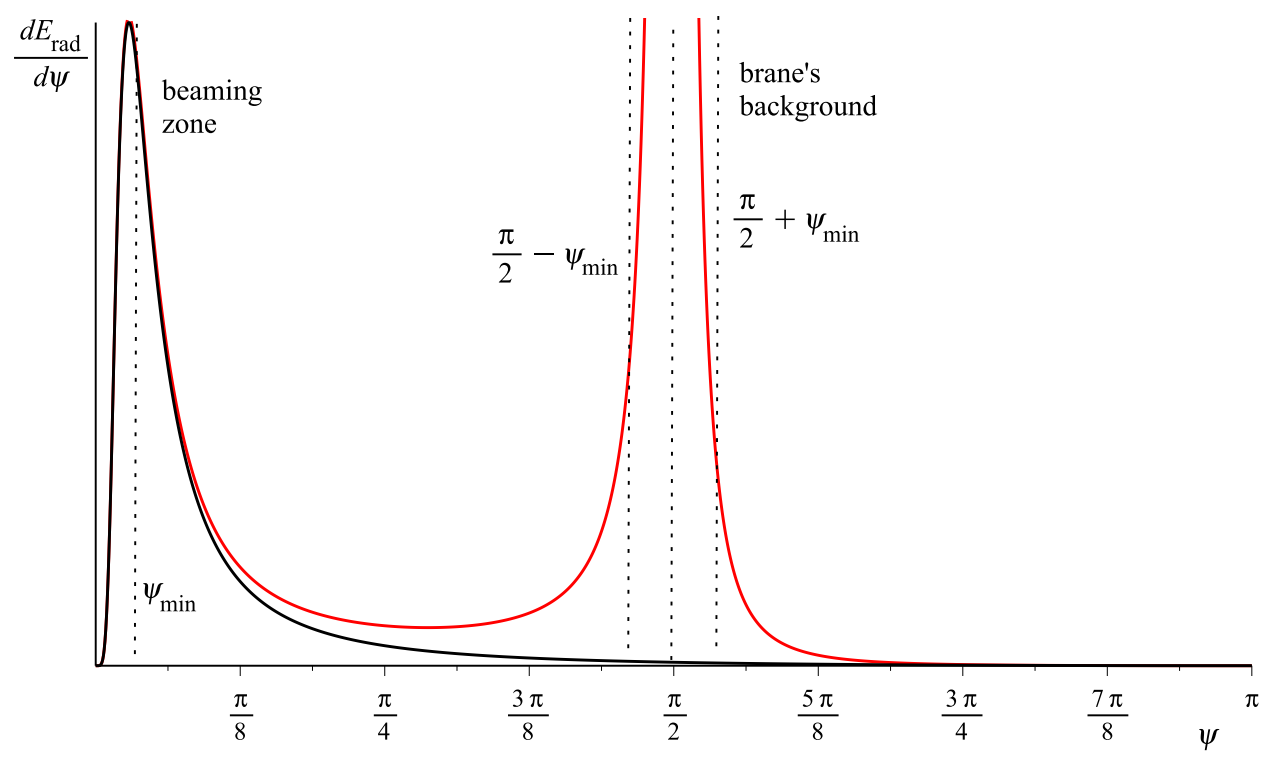

Figure 5. The total angular distribution of the emitted radiation for full source (red) and reduced one, with no brane excitation (black) for $D=5, \gamma=15$.

$\mathbb{Z}_{2}$-symmetric case. When both half-spaces of the brane hyperplane are equivalent, the brane excitation is absent:

$$
\Phi=0 .
$$

Thus the brane contribution to the total radiation amplitude is given by: ${ }^{10}$

$$
\left.{ }^{1} T_{z}^{*}(k) \equiv{ }^{1} T_{z}(k)\right|_{\Phi=0}=-\sqrt{\frac{D-3}{D-2}} \frac{\varkappa^{2} \mu \mathcal{E}}{2 \omega^{2}} \frac{v \sin ^{2} \psi}{1+\gamma^{2} v^{2} \sin ^{2} \psi}\left[\gamma^{2} v^{2}-\frac{1}{D-2}\right] .
$$

Thereby in this case the amplitude does not blow up at $\psi=\pi / 2$ and the angular distribution is finite. The characteristic plot with/without counting of $\Phi$ is shown on figure 5 . One sees that the reduction of $\Phi$ eliminates the infinite brane-motivated background at $\psi \sim \pi / 2$.

The total angular distribution may be integrated for $D \geqslant 6$ in the ultra-relativistic case. ${ }^{11}$ For $\gamma \gg 1$ one obtains:

$$
\left.\tau_{z}^{*}(k)\right|_{\psi \sim 1, \Phi=0, \gamma \gg 1}=\sqrt{\frac{D-3}{D-2}} \frac{\varkappa^{2} \mu \mathcal{E}}{\omega^{2}} \frac{1 / 2}{1-v \cos \psi} .
$$

The plot illustrating this approximation, is given on figure 7. Squaring of (6.21) and substituting it into (6.2) leads to the integrals $V_{2}^{D-3}$ which for $D>6$ turn out to be "nonbeamed" and for $D=6$ logarithmic, ${ }^{12}$ thus taking help of (A.7) this yields:

$$
E_{\mathrm{rad}}^{*}=\frac{1}{2^{7} \pi^{D / 2}} \frac{(D-3) \Gamma\left(\frac{D-6}{2}\right)}{(D-2)(D-5) \Gamma(D-4)}\left(\varkappa^{3} \mu \mathcal{E}\right)^{2} \omega_{\max }^{D-5} \quad D \geqslant 7,
$$

with $\omega_{\max }$ discussed above (6.10).

\footnotetext{
${ }^{10}$ The amplitude, emitted energy and radiation efficiency, corresponding to this mirror case, will be denoted with uppercase star.

${ }^{11}$ Remarkably, the validity of the low-angle approximation is extended to the angle region of order $\mathcal{O}(1)$, as it shown on figure 6 .

${ }^{12}$ The result reproduces (6.16) with logarithmic precision.
} 


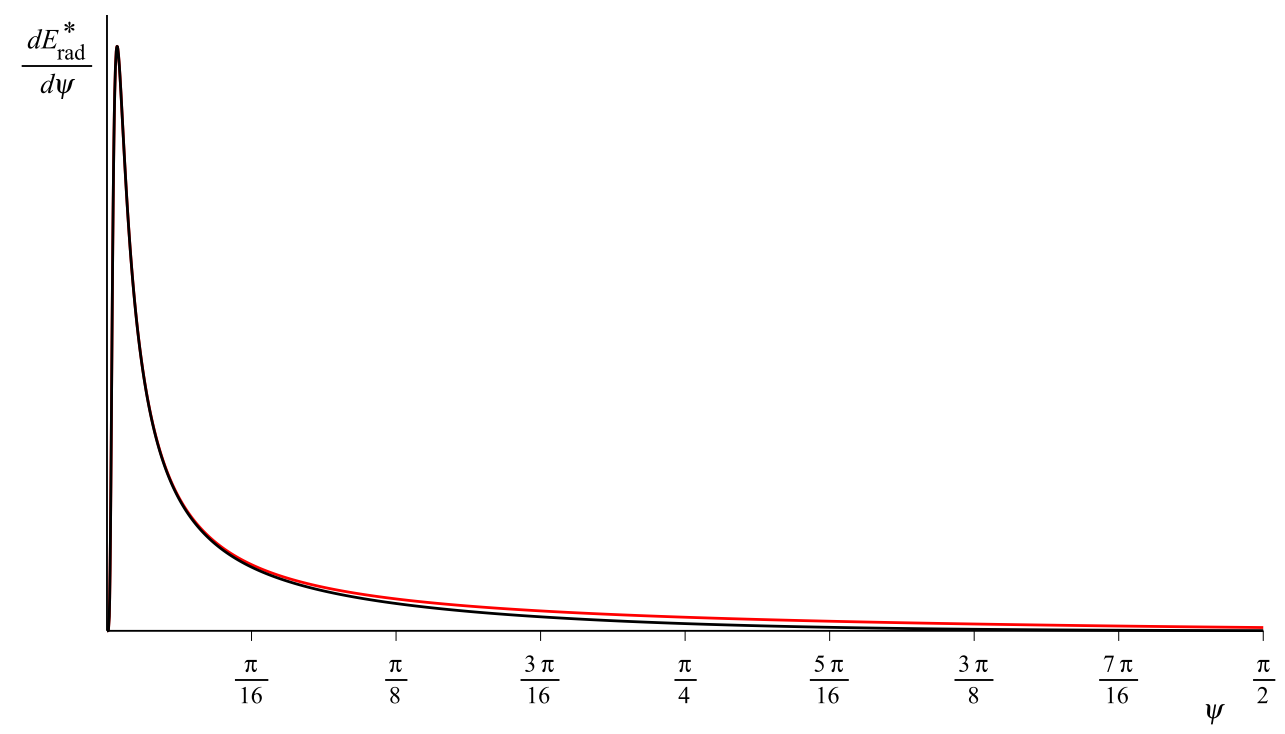

Figure 6. The reduced angular distribution of the emitted radiation (red) versus the approximation by formula (5.14) (black) for $D=6, \gamma=150$. For higher values of $\gamma$ two curves are more close to each other.

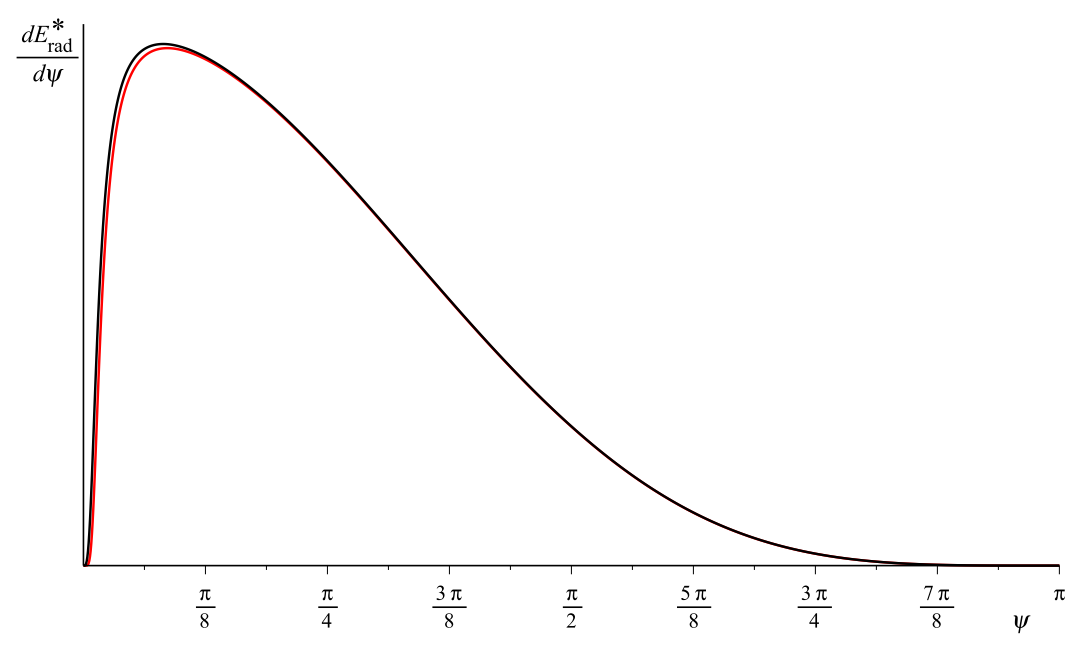

Figure 7. The reduced angular distribution of the emitted radiation (red) versus the approximation by formula (6.21) (black) for $D=7, \gamma=30$. For higher values of $\gamma$ two curves are more close to each other.

The formula (6.22) obtained for an ultrarelativistic particle, does not contain "free" Lorentz factor. Also, the angular cut-off at small angles is not required. Thus in higher dimensions there is no strong enhancement of the emitted radiation in the ultrarelativistic case. Omitting the brane's blow-up, the dependence of the emitted energy upon the speed of piercing particle is shown on figure 8 . 


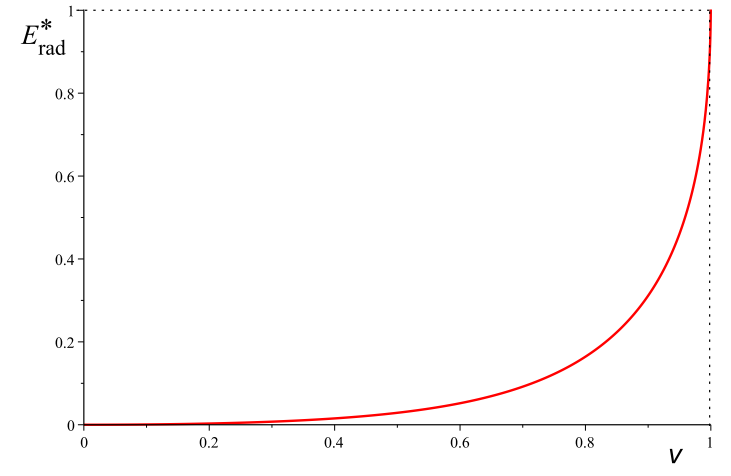

(a)

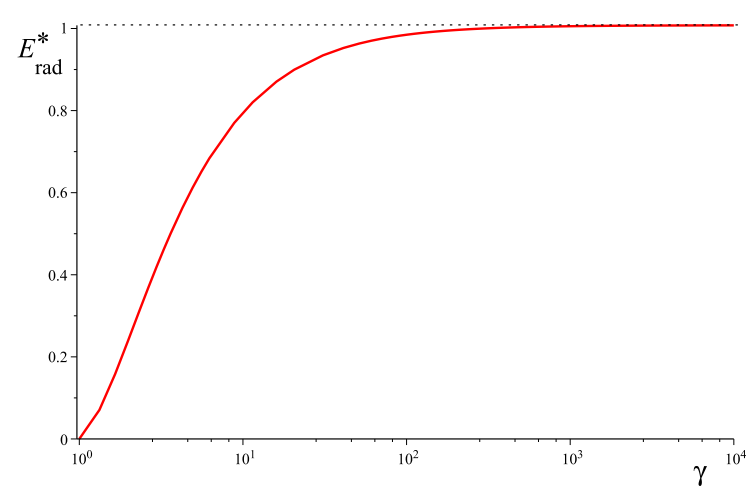

(b)

Figure 8. The emitted radiation against the speed of particle $(a)$ and Lorentz factor (in logarithmic mode) (b) in $D=7$ dimensions, normalized by the maximal value given by eq. (6.22).

The radiation efficiency is estimated as:

$$
\epsilon^{*} \sim\left(\frac{r_{\mathcal{E}}}{L}\right)^{2} \ln \frac{L}{r_{\mathcal{E}}}, D=6 \quad \epsilon^{*} \sim\left(\frac{r_{\mathcal{E}}}{L}\right)^{2}, D>6 .
$$

To summarize, the total efficiency of radiation can conveniently be expressed through the ratio of the beamed part to the brane contribution $\chi \equiv E_{\mathrm{rad}}^{*} / E_{\mathrm{br}}$ giving

$$
\epsilon \simeq \epsilon^{*} \theta(\chi-1)+\frac{\epsilon^{*}}{\chi} \theta(1-\chi)
$$

where $\theta(x)$ is the Heaviside step-function. In four and five dimensions the beamed radiation dominates, while in higher dimensions the most of radiation is emitted at large angles.

\section{Conclusions}

In this paper we investigated new mechanism of gravitational radiation from DWs, called PGR, due to their collisions with surrounding particles which perforate DWs and pass through. Within our model the DW-particle interaction was assumed to be purely gravitational and small, so the perturbative scheme on the Minkowski background is applicable. The gravitational force in this system is repulsive, so the particle must have the momentum transverse to the wall large enough in order to overcome repulsion and to perforate the wall. In plasma with some velocity distribution such particles form a layer whose size depends on their velocity; this size becomes large in the ultrarelativistic limit and for massless particles.

In the perturbation theory, gravitational radiation arises in the second, post-linear order. This is similar to perturbative treatment of gravitational bremsstrahlung under relativistic collisions of point particles, but in our case situation is more complicated since the wall is an extended object with an intrinsic dynamics due to tension. Another difference is that the force between the particle and the wall does not fall off with distance, so 
there are no asymptotically free states. So to calculate gravitational radiation in such a collision we had to resolve some conceptual and technical problems. One problem is failure of the traditional theory of radiation based on the notion of the wave zone which is absent in our case. So we had to reconsider formula for gravitational radiation without recurring to asymptotic conditions. The second problem is that, due to absence of free asymptotic states, perturbative description of gravity is restricted to certain distances around the wall, while the formal expansions in terms of gravitational coupling constant require considering in zero order an infinite motion of the free particle. Also, the point particle is not a good approximation itself, since any mass has an associated gravitational radius. Similarly, physical DW has a finite width, while we used the Nambu-Goto action. These oversimplifications allowed us to compute the radiation amplitudes analytically at the expense of the infrared and the ultraviolet divergences of the spectra, as well as (collinear) divergences in the angular distribution in the limit of massless particle. So we had to perform an additional analysis to motivate the choice of cutoffs needed to extract finite answers from the perturbation theory.

Due to the symmetry of the problem, the emitted radiation is polarized in single tensorial state, responsible for the bulk emission of GW. The radiation amplitude consists of the contribution due to the particle, to the DW and to gravitational stresses. The total radiation amplitude is factorized into the product of the frequency part and the angular part depending upon the single angle. The frequency factor $\omega^{-2}$ has an infrared blow-up, proper for the particles collisions [62] in general, though stronger. The angular part has divergence corresponding to gravitons emitted along the brane which is absent in the case of two mirror particles. In the non-relativistic limit the particle and stress contributions vanish, so only the brane contribution remains. For an ultrarelativistic particle the radiation amplitude in the forward direction is damped by two powers of $\gamma$ with respect to the particle term only due to destructive interference with the stress tensor contribution. Despite this, the radiation amplitude has a peak at $\psi \sim 1 / \gamma$ (with the same order of half-width), proper to radiation from fast particles (bremsstrahlung), with magnitude $\sim \gamma^{2}$.

The angular-frequency distribution of the emitted radiation, obtained after squaring the total amplitude and adding the dimension-dependent phase-volume factor, have the following features. The frequency distribution scales as $\sim \omega^{D-6}$ in the entire spectrum and therefore has infrared divergence in four dimensions, the ultraviolet divergence in higher dimensions, and both of them for $D=5$. All of them are removed by the appropriate cutoffs which may depend upon the particular DW model. The amplitude peak, discussed above, causes the beaming of the emitted gravitons, with characteristic cone angle $1 / \gamma$, as it common for fast particles. Such a beaming is realized in four and five dimensions, that reflects the corresponding enhancement of the emitted energy as gamma-factors in numerator. In the massless limit one uses the cutoff related with the applicability of linearized fields. However, in higher dimensions the dominant region of angular distribution shifts to the large angles, due to volume factor of the phase space. In this case the radiation tangentto-brane becomes dominant even with cutoff imposing. Thus in higher dimensions there is no strong enhancement of the emitted energy for relativistic/ultrarelativistic velocities, as well as no beaming of the emitted waves. 
In the massless limit the radiation amplitude has no peak and blows up as $\psi$ approaches zero. However, the resulting angular amplitude scales as $\sin ^{D-7} \psi$ and regular for $D>6$. The cutoff imposing solves this divergence at $D=4 \ldots 6$ in the same manner like for ultrarelativistic case with finite Lorentz factor exceeding the effective "cutoff" factor $\gamma_{*}$. In the non-relativistic limit the radiation due to the brane deflection dominates. Omitting the brane's influence, the radiation is roughly isotropic. The radiation efficiency depends on the braneworld model and dimensionality. In higher dimensions with infinite-sized brane it is found to be of order $\ln \gamma / \gamma$ or less, where for very high Lorentz factor one has to substitute it by effective $\gamma_{*}=L / r_{\mathcal{E}} \gg 1$.

In this paper we did not consider realistic cosmological applications: this would require different setting of the whole problem. But from our results it is clear that this mechanism may be relevant in the lower-frequency spectrum of the relic gravitons. Mention that the graviton spectrum from collapsing unstable domain walls is not divergent in the infrared but has maximum at some finite frequency [15]. Also, our mechanism is universal and applicable to stable DWs as well.

\section{Acknowledgments}

The work was supported by the Russian Foundation of Fundamental Research under the project 17-02-01299a. DG also acknowledges the Russian Government Program of Competitive Growth of the Kazan Federal University.

\section{A Angular integration}

Here we compute the angular integrals of the generic form

$$
V_{m}^{n}=\int_{0}^{\pi} \frac{\sin ^{n} \psi}{(1-v \cos \psi)^{m}} d \psi
$$

with integers $m, n$, and estimate them in the leading order in $\gamma \gg 1$. Making use of the formula [70]

$$
\int_{0}^{\pi} \frac{\sin ^{2 \nu-1} \theta}{(a-b \cos \theta)^{m}} d \theta=(2 / b)^{\nu-1 / 2} \sqrt{\pi} \Gamma(\nu)\left(a^{2}-b^{2}\right)^{\frac{2(\nu-m)-1}{4}} P_{\nu-m-1 / 2}^{1 / 2-\nu}\left(\frac{a}{\sqrt{a^{2}-b^{2}}}\right),
$$

valid for any real $a>|b|$, and $\operatorname{Re} \nu>0$, we express the result in terms of the associated Legendre function of the first kind $P_{\nu}^{\mu}(z)$. In our case $a=1, b=v$, so:

$$
V_{m}^{n}=(2 / v)^{n / 2} \sqrt{\pi} \Gamma\left(\frac{n+1}{2}\right) \gamma^{-n / 2+m} P_{n / 2-m}^{-n / 2}(\gamma)
$$

In non-relativistic limit $v \ll 1$ we start directly from (A.1) and expand it in powers of $v$. Integration over $\psi$ gives

$$
V_{m}^{n}=\int_{0}^{\pi}(1+m v \cos \psi) \sin ^{n} \psi d \psi+\mathcal{O}\left(v^{2}\right)=\frac{\sqrt{\pi} \Gamma\left(\frac{n+1}{2}\right)}{\Gamma\left(\frac{n+2}{2}\right)}+\mathcal{O}\left(v^{2}\right) .
$$


For $\gamma \gg 1$ one can use the asymptotic formula [69]:

$$
P_{\rho}^{\lambda}(\gamma)=\left(\frac{\Gamma(-\rho-1 / 2)}{2^{\rho+1} \sqrt{\pi} \Gamma(-\rho-\lambda)} \gamma^{-\rho-1}+\frac{2^{\rho} \Gamma(\rho+1 / 2)}{\sqrt{\pi} \Gamma(\rho-\lambda+1)} \gamma^{\rho}\right)\left(1+O\left(\gamma^{-2}\right)\right) .
$$

For $2 m>n+1$ the leading power of $\gamma$ comes from the first term in parenthesis of (A.5), thus retaining it one finds

$$
V_{m}^{n}=\frac{2^{m-1} \Gamma\left(\frac{n+1}{2}\right) \Gamma\left(m-\frac{n+1}{2}\right)}{\Gamma(m)} \gamma^{2 m-n-1} .
$$

Being applied to the computation of radiation flux, this type corresponds to beamed emission inside the spatial cone with characteristic angle of order $\mathcal{O}\left(\gamma^{-1}\right)$. Beyond this cone the integrand in (A.1) decreases rapidly. An alternative derivation of this answer can be found in appendix of [75].

For $2 m<n+1$ the behavior of Legendre function is governed by the second term in parenthesis of (A.5); thus one obtains

$$
V_{m}^{n}=\frac{2^{n-m} \Gamma\left(\frac{n+1}{2}\right) \Gamma\left(\frac{n+1}{2}-m\right)}{\Gamma(n-m+1)} .
$$

In the borderline case $2 m=n+1$ the behavior of the integral is logarithmic. Indeed, inserting the expansion (5.10) into (A.1) one integrates $1 / \psi$ from $\mathcal{O}\left(\gamma^{-1}\right)$ to $\mathcal{O}(1)$. Both terms in (A.5) become actual, hence representing $n=2 m-1+\epsilon$, we take the well-defined limit $\epsilon \rightarrow 0$, to obtain an asymptotic in this transition case:

$$
V_{m}^{2 m-1}=2^{m}\left[\ln 2 \gamma-H_{m-1}\right]+\mathcal{O}\left(\gamma^{-2}\right)
$$

where $H_{n}=\sum_{k=1}^{n} k^{-1}$ stands for the $n$-th harmonic number. It has to be pointed out that for contemporarily reasonable values of the Lorentz factor $\gamma \gg 1$, the $\mathcal{O}(1)$-term is comparable with $\mathcal{O}(\ln \gamma)$-term. This justifies the presence of non-logarithmic term here. In the special cases of interest here, with $m=2$ and 6 , integrals of this type are given by

$$
V_{2}^{3}=4(\ln 2 \gamma-1), \quad V_{6}^{11}=64\left(\ln 2 \gamma-\frac{137}{60}\right) .
$$

Furthermore, the generalization of angular integrals of the form

$$
V_{m_{1}, m_{2}}^{n}=\int_{0}^{\pi} \frac{\sin ^{n} \psi}{(1-v \cos \psi)^{m_{1}}(1+v \cos \psi)^{m_{2}}} d \psi
$$

in UR limit can be reduced into the case of single $V_{m}^{n}$ if $m \equiv \max \left\{m_{1}, m_{2}\right\}>(n+1) / 2$. In this case the domination domain is $0 \ldots \mathcal{O}(1 / \gamma)$ if $m=m_{1}, \pi-\mathcal{O}(1 / \gamma) \ldots \pi$ if $m=m_{2}$ and both of them if $m=m_{1}=m_{2}$. The leading in $\gamma$ order thereby is given by

$$
V_{m_{1}, m_{2}}^{n}= \begin{cases}2^{-m_{2}} V_{m_{1}}^{n}, & m_{1}>m_{2} ; \\ 2^{-m+1} V_{m}^{n}, & m_{1}=m_{2} ; \\ 2^{-m_{1}} V_{m_{2}}^{n}, & m_{1}<m_{2} .\end{cases}
$$

where $V_{m_{1}}^{n}$ and $V_{m_{2}}^{n}$ are given by (A.6). For arbitrary $v$ or if $m \leqslant(n+1) / 2$, the integrals of this type can be computed numerically. 


\section{B Applicability of the perturbation theory}

The linearized field generated by the DW approximately coincides with the full non-linear solution iff the bulk distance is small with respect to the brane-curvature radius, the latter is given by

$$
L \equiv\left(\varkappa^{2} \mu\right)^{-1} .
$$

In order to justify the correspondence of the linearized field generated by the particle one has to take into account the motion. In the particle rest frame the metric generated by the particle, is the Schwarzschild - Tangherlini one: in the isotropic coordinates $\left(\tilde{t}, \tilde{x}^{1}, \ldots, \tilde{x}^{D-1}\right)$ it is given by

$$
\mathrm{d} s^{2}=\frac{\left[1-\left(\varrho_{g} / \varrho\right)^{D-3}\right]^{2}}{\left[1+\left(\varrho_{g} / \varrho\right)^{D-3}\right]^{2}} \mathrm{~d} \tilde{t}^{2}-\left[1+\left(\frac{\varrho g}{\varrho}\right)^{D-3}\right]^{\frac{4}{D-3}} \delta_{i j} \mathrm{~d} \tilde{x}^{i} \mathrm{~d} \tilde{x}^{j},
$$

where $\varrho \equiv\left(\delta_{i j} \tilde{x}^{i} \tilde{x}^{j}\right)^{1 / 2}$ and $i, j$ run from 1 to $D-1$. Here $\varrho>\varrho_{g}$ and $\varrho_{g}$ stands for the isotropic-coordinate gravitational radius, which is given by

$$
\varrho_{g}=\frac{1}{\sqrt{\pi}}\left[\frac{2 \Gamma\left(\frac{D-1}{2}\right) G m}{D-2}\right]^{\frac{1}{D-3}} .
$$

Now we transform (B.1) to our Lab frame which is the unexcited-brane rest frame, with coordinates $\left(t, x^{1}, \ldots, x^{D-2}, z\right)$ used in the main text. Applying the Lorentz boost in $z$-direction $t=\gamma\left(\tilde{t}+v \tilde{x}^{D-1}\right), z=\gamma\left(\tilde{x}^{D-1}+v \tilde{t}\right), x^{i}=\tilde{x}^{i}$, and introducing the dimensionless factor

with

$$
A \equiv\left(\frac{\varrho g}{\varrho}\right)^{D-3}=\frac{2 m G \Gamma\left(\frac{D-1}{2}\right)}{(D-2)(\sqrt{\pi} \varrho)^{D-3}}
$$

$$
\varrho=\sqrt{\gamma^{2}(z-v t)^{2}+r^{2}}, \quad r^{2}=\delta_{i j} x^{i} x^{j} .
$$

the line element (B.1) reads

$$
\mathrm{d} s^{2}=(1+A)^{\frac{4}{D-3}} \mathrm{~d} s_{\mathcal{M}^{+}}^{2}\left[\left(\frac{1-A}{1+A}\right)^{2}-(1+A)^{\frac{4}{D-3}}\right] \gamma^{2}(\mathrm{~d} t-v \mathrm{~d} z)^{2},
$$

where $\mathrm{d} s_{\mathcal{M}}^{2}=\eta_{M N} \mathrm{~d} x^{M} \mathrm{~d} x^{N}$ is Minkowskian metric.

Thereby

$$
A=\frac{2 G \mathcal{E} \Gamma\left(\frac{D-1}{2}\right)}{(D-2)(\sqrt{\pi} \varrho)^{D-3} \gamma} \ll 1
$$

is sufficient for validity of a Taylor expansion: retaining the first order, one obtains

$$
\mathrm{d} s^{2}=\left[1+\frac{4 A}{D-3}\right] \mathrm{d} s_{\mathcal{M}}^{2}-\frac{4(D-2) A}{D-3} \gamma^{2}(\mathrm{~d} t-v \mathrm{~d} z)^{2} .
$$

The non-zero components of this metric are:

$$
\begin{aligned}
g_{00} & =1-\frac{4 A}{D-3}\left[(D-2) \gamma^{2}-1\right] & g_{0 z} & =\frac{4(D-2) A}{D-3} \gamma^{2} v \\
g_{z z} & =-1-\frac{4 A}{D-3}\left[(D-2) \gamma^{2} v^{2}+1\right] & g_{x_{k} x_{k}} & =-1-\frac{4 A}{D-3}
\end{aligned}
$$


which after substitution of $\varkappa^{2}=16 \pi G$ exactly correspond to those ones of the linearized solution $\bar{h}_{M N}(2.12)$

$$
\bar{h}_{M N}(x)=-\frac{\varkappa m \Gamma\left(\frac{D-3}{2}\right)}{4 \pi^{\frac{D-1}{2}} \varrho^{D-3}}\left(u_{M} u_{N}-\frac{1}{D-2} \eta_{M N}\right) .
$$

Thereby the cut-offs due the moving particle are

$$
r_{\min } \simeq r_{g}, \quad z_{\min } \simeq r_{g} / \gamma .
$$

Introducing the energy-associated gravitational radius $r_{\mathcal{E}}$ as

$$
r_{\mathcal{E}}=\frac{1}{\sqrt{\pi}}\left[\frac{2 \Gamma\left(\frac{D-1}{2}\right) G \mathcal{E}}{D-2}\right]^{\frac{1}{D-3}}
$$

which is assumed to be constant independently of $\gamma$, one observes that when tending to the massless limit, the minimal-length cutoffs due to the moving particle vanish as $r_{\text {min }} \sim \gamma^{-1 /(D-3)}, z_{\text {min }} \sim \gamma^{-(D-2) /(D-3)}$.

If we live on the brane, piercing by the bulk black hole will excite explosive branons whose energy can be transformed to matter on the brane. To calculate such effects on has, however, to apply different techniques.

Open Access. This article is distributed under the terms of the Creative Commons Attribution License (CC-BY 4.0), which permits any use, distribution and reproduction in any medium, provided the original author(s) and source are credited.

\section{References}

[1] I. Yu. Kobsarev, L.B. Okun and Ya. B. Zeldovich, Spontaneous CP-violation and cosmology, Phys. Lett. B 50 (1974) 340 [inSPIRE].

[2] Ya. B. Zeldovich, I. Yu. Kobzarev and L.B. Okun, Cosmological Consequences of the Spontaneous Breakdown of Discrete Symmetry, Zh. Eksp. Teor. Fiz. 67 (1974) 3 [inSPIRE].

[3] T.W.B. Kibble, Topology of Cosmic Domains and Strings, J. Phys. A 9 (1976) 1387 [INSPIRE].

[4] A. Vilenkin, Gravitational Field of Vacuum Domain Walls and Strings, Phys. Rev. D 23 (1981) 852 [INSPIRE].

[5] A. Vilenkin, Cosmic Strings and Domain Walls, Phys. Rept. 121 (1985) 263 [InSPIRE].

[6] A. Vilenkin and E.P.S. Shellard, Cosmic Strings and other Topological Defects Cambridge, University Press, U.K. (2000).

[7] M. Khlopov, Fundamentals of Cosmic Particle Physics, Cambridge International Science Publishing, (2012), https://doi.org/10.1007/978-1-907343-72-8.

[8] T. Hiramatsu, M. Kawasaki and K. Saikawa, Gravitational Waves from Collapsing Domain Walls, JCAP 05 (2010) 032 [arXiv: 1002.1555] [INSPIRE].

[9] M. Kawasaki and K. Saikawa, Study of gravitational radiation from cosmic domain walls, JCAP 09 (2011) 008 [arXiv: 1102.5628] [INSPIRE]. 
[10] T. Hiramatsu, M. Kawasaki and K. Saikawa, On the estimation of gravitational wave spectrum from cosmic domain walls, JCAP 02 (2014) 031 [arXiv:1309.5001] [INSPIRE].

[11] N. Kitajima and F. Takahashi, Gravitational waves from Higgs domain walls, Phys. Lett. B $\mathbf{7 4 5}$ (2015) 112 [arXiv:1502.03725] [INSPIRE].

[12] K. Kadota, M. Kawasaki and K. Saikawa, Gravitational waves from domain walls in the next-to-minimal supersymmetric standard model, JCAP 10 (2015) 041 [arXiv:1503.06998] [INSPIRE].

[13] K. Nakayama, F. Takahashi and N. Yokozaki, Gravitational waves from domain walls and their implications, Phys. Lett. B 770 (2017) 500 [arXiv:1612.08327] [INSPIRE].

[14] T. Krajewski, Z. Lalak, M. Lewicki and P. Olszewski, Domain walls and gravitational waves in the Standard Model, JCAP 12 (2016) 036 [arXiv:1608.05719] [INSPIRE].

[15] K. Saikawa, A review of gravitational waves from cosmic domain walls, Universe 3 (2017) 40 [arXiv: 1703.02576] [INSPIRE].

[16] L. Randall and R. Sundrum, An alternative to compactification, Phys. Rev. Lett. 83 (1999) 4690 [hep-th/9906064] [INSPIRE].

[17] D. Langlois, Brane cosmology: An introduction, Prog. Theor. Phys. Suppl. 148 (2003) 181 [hep-th/0209261] [INSPIRE].

[18] N. Tanahashi and T. Tanaka, Black holes in braneworld models, Prog. Theor. Phys. Suppl. 189 (2011) 227 [arXiv: 1105.2997] [INSPIRE].

[19] V.P. Frolov, M. Snajdr and D. Stojkovic, Interaction of a brane with a moving bulk black hole, Phys. Rev. D 68 (2003) 044002 [gr-qc/0304083] [INSPIRE].

[20] A. Chamblin and D.M. Eardley, Puncture of gravitating domain walls, Phys. Lett. B 475 (2000) 46 [hep-th/9912166] [INSPIRE].

[21] D. Stojkovic, K. Freese and G.D. Starkman, Holes in the walls: Primordial black holes as a solution to the cosmological domain wall problem, Phys. Rev. D 72 (2005) 045012 [hep-ph/0505026] [INSPIRE].

[22] A. Flachi and T. Tanaka, Branes and Black holes in Collision, Phys. Rev. D 76 (2007) 025007 [hep-th/0703019] [INSPIRE].

[23] V.P. Frolov, Merger Transitions in Brane-Black-Hole Systems: Criticality, Scaling and Self-Similarity, Phys. Rev. D 74 (2006) 044006 [gr-qc/0604114] [InSPIRE].

[24] V.P. Frolov, D.V. Fursaev and D. Stojkovic, Interaction of higher dimensional rotating black holes with branes, Class. Quant. Grav. 21 (2004) 3483 [gr-qc/0403054] [INSPIRE].

[25] V.P. Frolov, D.V. Fursaev and D. Stojkovic, Rotating black holes in brane worlds, JHEP 06 (2004) 057 [gr-qc/0403002] [INSPIRE].

[26] V.G. Czinner and A. Flachi, Thickness perturbations and topology change transitions in brane: Black hole systems, Phys. Rev. D 80 (2009) 104017 [arXiv:0908.2957] [INSPIRE].

[27] V.G. Czinner, Thick brane solutions and topology change transition on black hole backgrounds, Phys. Rev. D 82 (2010) 024035 [arXiv:1006.4424] [INSPIRE].

[28] A. Flachi, O. Pujolàs, M. Sasaki and T. Tanaka, Critical escape velocity of black holes from branes, Phys. Rev. D 74 (2006) 045013 [hep-th/0604139] [INSPIRE]. 
[29] A. Flachi and T. Tanaka, Escape of black holes from the brane, Phys. Rev. Lett. 95 (2005) 161302 [hep-th/0506145] [INSPIRE].

[30] A. Flachi, O. Pujolàs, M. Sasaki and T. Tanaka, Black holes escaping from domain walls, Phys. Rev. D 73 (2006) 125017 [hep-th/0601174] [InSPIRE].

[31] D.V. Gal'tsov, E. Yu. Melkumova and S. Zamani-Mogaddam, Nambu-Goldstone explosion under brane perforation, JETP Lett. 92 (2010) 276 [arXiv:1012.2841] [INSPIRE].

[32] D.V. Gal'tsov, E. Yu. Melkumova and P.A. Spirin, Perforation of a domain wall by a point mass, Phys. Rev. D 89 (2014) 085017 [arXiv:1312.7760] [InSPIRE].

[33] D.V. Gal'tsov, E. Yu. Melkumova and P.A. Spirin, Energy-momentum balance in a particle domain wall perforating collision, Phys. Rev. D 90 (2014) 125024 [arXiv:1411.0122] [INSPIRE].

[34] D. Gal'tsov, E. Melkumova and P. Spirin, Branestrahlung: radiation in the particle-brane collision, Phys. Rev. D 93 (2016) 045018 [arXiv:1512.04607] [inSPIRE].

[35] D.V. Galtsov, Radiation reaction in various dimensions, Phys. Rev. D 66 (2002) 025016 [hep-th/0112110] [INSPIRE].

[36] D.V. Gal'tsov and P.A. Spirin, Radiation reaction in curved even-dimensional spacetime, Grav. Cosmol. 13 (2007) 241.

[37] D. Gal'tsov, P. Spirin and S. Staub, Radiation reaction in curved space-time: Local method, in Gravitation and Astrophysics, J.M. Nester, C.-M. Chen and J.-P. Hsu eds., World Scientific, Singapore, (2006), p. 345, gr-qc/0701004 [INSPIRE].

[38] F. Azzurli and K. Lechner, The Lienard-Wiechert field of accelerated massless charges, Phys. Lett. A 377 (2013) 1025 [arXiv:1212.3532] [INSPIRE].

[39] F. Azzurli and K. Lechner, Electromagnetic fields and potentials generated by massless charged particles, Annals Phys. 349 (2014) 1 [arXiv:1401.5721] [INSPIRE].

[40] A. Tolish and R.M. Wald, Retarded Fields of Null Particles and the Memory Effect, Phys. Rev. D 89 (2014) 064008 [arXiv: 1401.5831] [INSPIRE].

[41] T. Kinoshita, Mass singularities of Feynman amplitudes, J. Math. Phys. 3 (1962) 650 [INSPIRE].

[42] T.D. Lee and M. Nauenberg, Degenerate Systems and Mass Singularities, Phys. Rev. 133 (1964) B1549 [INSPIRE].

[43] M. Nauenberg, The Zero Mass Limit of a Charged Particle in Quantum Electrodynamics, Acta Phys. Austriaca Suppl. 2 (1965) 234 [INSPIRE].

[44] S. Weinberg, Infrared photons and gravitons, Phys. Rev. 140 (1965) B516 [InSPIRE].

[45] D.V. Gal'tsov, Synchrotron radiation from massless charge, Phys. Lett. B 747 (2015) 400 [arXiv: 1505.06775] [INSPIRE].

[46] A. Tolish, L. Bieri, D. Garfinkle and R.M. Wald, Examination of a simple example of gravitational wave memory, Phys. Rev. D 90 (2014) 044060 [arXiv: 1405.6396] [InSPIRE].

[47] L. Bieri and D. Garfinkle, Perturbative and gauge invariant treatment of gravitational wave memory, Phys. Rev. D 89 (2014) 084039 [arXiv:1312.6871] [InSPIRE].

[48] J. Winicour, Global aspects of radiation memory, Class. Quant. Grav. 31 (2014) 205003 [arXiv: 1407.0259] [INSPIRE]. 
[49] L. Bieri, D. Garfinkle and S.-T. Yau, Gravitational Waves and Their Memory in General Relativity, arXiv:1505.05213 [INSPIRE].

[50] A. Strominger and A. Zhiboedov, Gravitational Memory, BMS Supertranslations and Soft Theorems, JHEP 01 (2016) 086 [arXiv:1411.5745] [INSPIRE].

[51] R. Gregory, V.A. Rubakov and S.M. Sibiryakov, Brane worlds: The gravity of escaping matter, Class. Quant. Grav. 17 (2000) 4437 [hep-th/0003109] [INSPIRE].

[52] W. Mueck, K.S. Viswanathan and I.V. Volovich, Geodesics and Newton's law in brane backgrounds, Phys. Rev. D 62 (2000) 105019 [hep-th/0002132] [INSPIRE].

[53] S.L. Dubovsky, V.A. Rubakov and P.G. Tinyakov, Brane world: Disappearing massive matter, Phys. Rev. D 62 (2000) 105011 [hep-th/0006046] [INSPIRE].

[54] M. Maziashvili, Dynamics of massive matter disappearance on the brane, Phys. Lett. B 627 (2005) 197 [hep-ph/0507103] [INSPIRE].

[55] M. Maziashvili, Particle escape into extra space, Phys. Lett. B 635 (2006) 36 [hep-ph/0512362] [INSPIRE].

[56] A. Friedland and M. Giannotti, Astrophysical bounds on photons escaping into extra dimensions, Phys. Rev. Lett. 100 (2008) 031602 [arXiv:0709.2164] [INSPIRE].

[57] J. Guven, Covariant perturbations of domain walls in curved space-time, Phys. Rev. D 48 (1993) 4604 [gr-qc/9304032] [INSPIRE].

[58] M. Bando, T. Kugo, T. Noguchi and K. Yoshioka, Brane fluctuation and suppression of Kaluza-Klein mode couplings, Phys. Rev. Lett. 83 (1999) 3601 [hep-ph/9906549] [InSPIRE].

[59] T. Kugo and K. Yoshioka, Probing extra dimensions using Nambu-Goldstone bosons, Nucl. Phys. B 594 (2001) 301 [hep-ph/9912496] [INSPIRE].

[60] Y. Burnier and K. Zuleta, Effective action of a five-dimensional domain wall, JHEP 05 (2009) 065 [arXiv: 0812.2227] [INSPIRE].

[61] J. Alexandre and D. Yawitch, Comments on branon dressing and the Standard Model, New J. Phys. 12 (2010) 043027 [arXiv: 0910.5150] [InSPIRE].

[62] S. Weinberg, Gravitation and Cosmology: Principles and Applications of the General Theory of Relativity, Wiley (1972).

[63] D.V. Galtsov, Yu. V. Grats and A.A. Matyukhin, Problem of bremsstrahlung in: The case of gravitational interaction, Sov. Phys. J. 23 (1980) 389 [INSPIRE].

[64] Y. Constantinou, D. Gal'tsov, P. Spirin and T.N. Tomaras, Scalar Bremsstrahlung in Gravity-Mediated Ultrarelativistic Collisions, JHEP 11 (2011) 118 [arXiv:1106.3509] [INSPIRE].

[65] D. Gal'tsov, P. Spirin and T.N. Tomaras, Gravitational bremsstrahlung in ultra-planckian collisions, JHEP 01 (2013) 087 [arXiv:1210.6976] [INSPIRE].

[66] K. Lechner, Electrodynamics of massless charged particles, J. Math. Phys. 56 (2015) 022901 [arXiv: 1405.4805] [INSPIRE].

[67] D.V. Gal'tsov, G. Kofinas, P. Spirin and T.N. Tomaras, Transplanckian bremsstrahlung and black hole production, Phys. Lett. B 683 (2010) 331 [arXiv:0908.0675] [INSPIRE].

[68] I.B. Khriplovich and E.V. Shuryak, Radiation emitted by an ultrarelativistic particle in a gravitational field, Zh. Eksp. Teor. Fiz. 65 (1973) 2137 [INSPIRE]. 
[69] I.S. Gradshteyn and I.M. Ryzhik, Table of Integrals, Series and Products, Academic Press, (1965).

[70] A.P. Prudnikov, Yu. A. Brychkov and O.I. Marichev, Integrals and Series, Vol. 1, Elementary Functions, Gordon \& Breach Sci. Publ., New York, U.S.A., (1986).

[71] V.S. Vladimirov, Equations of Mathematical Physics, Dekker, New York, U.S.A. (1971).

[72] V. Vladimirov and I. Petrova, Distributions en physique mathématique (in French), Moscow, URSS (2008).

[73] I.M. Gel'fand and G.E. Shilov, Generalized Functions, Vol. I, Academic Press, (1964).

[74] D.V. Galtsov, G. Kofinas, P. Spirin and T.N. Tomaras, Classical ultrarelativistic bremsstrahlung in extra dimensions, JHEP 05 (2010) 055 [arXiv: 1003.2982] [INSPIRE].

[75] Y. Constantinou and P. Spirin, Vector Bremsstrahlung by Ultrarelativistic Collisions in Higher Dimensions, JHEP 01 (2014) 111 [arXiv:1310.6065] [INSPIRE]. 